WSR C-RP-2004-00256

\title{
Savannah River Site Annual Meteorology Report for 2003 (U)
}

Westinghouse Savannah River Company

Savannah River Site

Aiken, SC 29808

Prepared for the U. S. Department of Energy under contract no. DE-AC09-96SR18500 
This document was prepared in conjunction with work accomplished under Contract No. DE-AC09-96SR18500 with the U. S. Department of Energy.

\section{DISCLAIMER}

This report was prepared as an account of work sponsored by an agency of the United States Government. Neither the United States Government nor any agency thereof, nor any of their employees, makes any warranty, express or implied, or assumes any legal liability or responsibility for the accuracy, completeness, or usefulness of any information, apparatus, product or process disclosed, or represents that its use would not infringe privately owned rights. Reference herein to any specific commercial product, process or service by trade name, trademark, manufacturer, or otherwise does not necessarily constitute or imply its endorsement, recommendation, or favoring by the United States Government or any agency thereof. The views and opinions of authors expressed herein do not necessarily state or reflect those of the United States Government or any agency thereof.

This report has been reproduced directly from the best available copy.

Available for sale to the public, in paper, from: U.S. Department of Commerce, National Technical Information Service, 5285 Port Royal Road, Springfield, VA 22161, phone: (800) 553-6847, fax: (703) 605-6900

email: orders@ntis.fedworld.gov

online ordering: http://www.ntis.gov/help/index.asp

Available electronically at http://www.osti.gov/bridge

Available for a processing fee to U.S. Department of Energy and its contractors, in paper, from: U.S. Department of Energy, Office of Scientific and Technical Information, P.O. Box 62, Oak Ridge, TN 37831-0062,

phone: (865)576-8401,

fax: (865)576-5728

email: $\underline{\text { reports@ adonis.osti.gov }}$ 
WSRC-RP-2004-00256

\title{
Savannah River Site Annual Meteorology Report for 2003 (U)
}

\author{
C. H. Hunter
}

Westinghouse Savannah River Company

Savannah River Site

Aiken, SC 29808

Prepared for the U. S. Department of Energy under contract no. DE-AC09-96SR18500 


\section{Contents}

$\begin{array}{ll}\text { Summary } & 1\end{array}$

$\begin{array}{ll}\text { Background } & 2\end{array}$

The General SRS Climate 2

Overview of Meteorological Monitoring at the 2

Savannah River Site

Data Sources for the 2003 Report 3

The SRS Climatology for $2003 \quad 5$

Overview

Temperature 5

Precipitation $\quad 6$

Atmospheric Moisture 6

Heat Stress $\quad 7$

Wind $\quad 7$

Barometric Pressure $\quad 7$

$\begin{array}{lc}\text { Solar Radiation } & 8\end{array}$

References $\quad 9$

$\begin{array}{ll}\text { Appendix A } & 32\end{array}$ 


\section{List of Tables}

Table 1. Means and Extremes for 2003

(a) Temperature and Precipitation

(b) Dew Point, Wet Bulb, Relative Humidity, and Wet Bulb Globe Temperature

(c) Wind Speed, Barometric Pressure, Solar Radiation, and Heating / Cooling Degree Days

Table 2. Monthly and Annual Average and Extreme Temperatures, 1973-2003

Table 3. Monthly and Annual Average and Extreme Rainfall, 1973-2003

Table 4. Monthly and Annual Rainfall, Manual Gauges

Table A Joint Occurrence Frequencies of Wind Direction Sector by Wind Speed Wind Speed Category, 2003
A.1 A Area Tower
A.2 C Area Tower
A.3 D Area Tower
A.4 F Area Tower
A.5 H Area Tower
A.6 K Area Tower
A.7 L Area Tower
A.8 P Area Tower
A.9 2m Level Central Climatology Tower
A.10 18m Level Central Climatology Tower
A.11 36m Level Central Climatology Tower
A.12 61m Level Central Climatology Tower
A.13 61m Level Central Climatology Tower, Winter
A.14 61m Level Central Climatology Tower, Spring
A.15 61m Level Central Climatology Tower, Summer
A.16 61m Level Central Climatology Tower, Fall 


\section{List of Figures}

Figure 1. Meteorological Monitoring Stations

Figure 2. Summary of Daily Data for 2003

Figure 3. Daily High and Low Temperatures for 2003

Figure 4. SRS Annual Average Temperature 1973-2003

Figure 5. SRS Monthly Average Temperature

Figure 6. Number of Freezing (<32F) and Sweltering ( $>90 F)$ Days

Figure 7. Daily Precipitation for 2003

Figure 8. SRS Annual Precipitation 1973-2003

Figure 9. SRS Monthly Precipitation

Figure 10. Daily High and Low Humidity for 2003

Figure 11. SRS Annual Average Humidity 1973-2003

Figure 12. SRS Monthly Average Minimum Humidity

Figure 13. Daily Average Wind Speed for 2003

Figure 14. Annual Wind Rose Plots for 2003, 61m Level

(a) A, C, D, and F Area Towers

(b) H, K, L, and P Area Towers

Figure 15. Annual Wind Rose Plots for 2003, Central Climatology Tower, All Levels

Figure 16. Seasonal Wind Rose Plots for 2003, Central Climatology Tower, 61m Level

Figure 17. Daily Average Barometric Pressure for 2003

Figure 18. Daily Total Solar Radiation for 2003 


\section{Summary}

Summaries of meteorological observations collected at the Savannah River Site in 2003 reveal a year that was unusually cool and wet. The annual rainfall of 61.2 inches was the third highest of all the years in a period of record that began in 1952. Higher amounts were recorded only in 1964 (73.5 in) and 1971 (68.2 in). Rainfall of 0.01 inch or more occurred on 119 days during the year.

Furthermore, the annual average temperature of 62.2 oF was the coldest of any year in an available record that dates to 1964. Cool and wet conditions were most pronounced in the spring and summer months. Unusually cold weather also occurred in January and December.
The coldest temperature for the year was 12.5 of (Jan 24) and the warmest temperature was 92.4 oF (Aug 27).

There were no significant occurrences of severe weather (ice/ snow, tornado, sustained high wind) during the year. The heavy rain that occurred on April 7 (3.5 inches) was due to an active stationary front over the area and strong southwesterly wind aloft. The remnants of Tropical Storm Bill produced 2.36 inches of rain on July 1 . Hurricane Isabelle, which struck the North Carolina coast mid September, did not have a significant affect on the SRS. A thunderstorm on May 3 produced a surface (4-meter) wind gust of 41.7 miles per hour. 


\section{Background}

\section{The General SRS Climate}

The Savannah River Site region has a humid subtropical climate characterized by relatively short, mild winters and long, warm, and humid summers (Oliver and Fairbridge, 1987).

Summer-like conditions typically last from May through September, when the area is frequently under the influence of the western extension of the semi-permanent Atlantic subtropical anticyclone (i.e. the 'Bermuda' high). Winds in summer are light and cold fronts generally remain well north of the area. Daily high temperatures during the summer months exceed $90^{\circ} \mathrm{F}$ on more than half of all days on the average. Scattered afternoon and evening thunderstorms are common.

The influence of the Bermuda high begins to diminish during the fall, resulting in lower humidity and more moderate temperatures. Average rainfall during the fall is usually the least of the four seasons.

In the winter months, mid-latitude low pressure systems and associated fronts often migrate through the region. As a result, conditions frequently alternate between warm, moist, subtropical air from the Gulf of Mexico region and cool, dry polar air. The Appalachian Mountains to the north and northwest of the SRS help to moderate the extremely cold temperatures associated with occasional outbreaks of Arctic air. Consequently, less than onethird of winter days have minimum temperatures below freezing on average, and days with temperatures below $20^{\circ} \mathrm{F}$ are infrequent. Measurable snowfall is rare.
Tornadoes and severe thunderstorms occur more frequently in spring than in other seasons of the year. Although spring weather is somewhat windy, temperatures are usually mild and humidity is relatively low.

\section{Overview of the Savannah River Site Meteorological M onitoring Program}

Meteorological data are collected at SRS from a network of nine primary monitoring stations (Fig. 1). Towers located adjacent to each of eight primary operations areas (A, C, D, F, H, K, L, and $\mathrm{P}$ areas) are equipped to measure wind direction, wind speed, temperature, and dew point at a height of 61 meters $(\mathrm{m})$ above ground. Temperature and dew point are also measured at $2 \mathrm{~m}$. A ninth tower near $\mathrm{N}$ Area, known as the Central Climatology site (CLM), is instrumented with wind, temperature, and dew point sensors at four levels: $2 \mathrm{~m}$ ( $4 \mathrm{~m}$ for wind), $18 \mathrm{~m}, 36 \mathrm{~m}$, and $61 \mathrm{~m}$. The CLM site is also equipped with an automated tipping bucket rain gauge, a barometric pressure sensor, and a solar radiometer near the tower at ground level. Data acquisition units at each station record a measurement from each instrument at 1second intervals. Every 15 minutes, the 1second data are processed to generate statistical summaries for each variable, including averages and instantaneous maxima, and the results uploaded to a relational database for archival. All aspects of the meteorological data collection program meet or exceed applicable regulatory criteria. Parker and Addis (1993) provide a complete description of the meteorological monitoring program at SRS.

Quality assurance of the data is conducted in two phases: an initial screening of recent 
data, followed by an in-depth review and final quality classification. The initial screening, performed twice daily by qualified instrument technicians, consists of a thorough examination of 15-minute data retrieved from the database, in conjunction with a summary of instrument diagnostics obtained from the local data acquisition units. Potential problems are noted in a daily checksheet and, as needed, data acquisition unit software is instructed to assign a quality control tag to data collected from questionable instruments. Quality tags are also set during periods of calibration and maintenance.

The second phase of the quality assurance process is conducted according to formal procedure (SRTC, 2003). Daily checksheets generated during the initial screening, tower-specific logbook entries, initial quality tags, and time series plots of related data are reviewed to determine a final quality status for each record. All records permanently archived in the data base are identified as good, fatal, intermittent, biased, or uncalibrated.

A major initiative to port all meteorological data base operations to a UNIX server was completed in 2003. The data base system had previously operated on a VMS computer cluster.

\section{Additional Measurements}

Additional precipitation measurements are collected from a network of 12 plastic wedge rain gauges across the SRS (See Fig. 1). These gauges are read manually by security personnel once per day, usually around $6 \mathrm{am}$. The daily data are reported to the SRS Atmospheric Technologies Center, reviewed to correct obvious flaws, and manually entered into an electronic data base table residing on the UNIX server.
Additional measurements of temperature and relative humidity are recorded from a station located in A-Area, adjacent to 773A. This station consists of a standard National Weather Service 'cotton region' instrument shelter. Data collected from this station are manually tabulated for archival as daily high and low values of temperature and relative humidity. Tabulated values are then entered into an electronic data base table on the UNIX server.

\section{Data Sources for the 2003 Report}

Data summaries for this report were chosen to give a general overview of climatological conditions for SRS.

Specific summaries are provided for:

- Ground level measurements of temperature $(2 \mathrm{~m})$, dew point temperature $(2 m)$, wind speed $(4 m)$, precipitation, barometric pressure, and solar radiation from CLM, as well as meteorological quantities derived from these primary data, such as relative humidity (temperature and dew point), wet bulb temperature (temperature and dew point), wet bulb globe temperature (temperature, dew point, wind speed, and solar radiation), and cooling/heating degree days (temperature).

- Joint occurrence frequencies of wind speed and direction from measurements taken at each of the four levels of the CLM tower and the $61 \mathrm{~m}$ level of the eight area towers.

- Precipitation from the network of manually-read rain gauges

A series of SAS software programs were used to extract the desired data sets from 
the data base and perform initial statistical processing on all records with a QA status of 'good'. More than $99 \%$ of the 2003 data used in this report met this criterion. Output from the initial processing was then imported into an Excel spreadsheet to generate the final summaries that are presented in the report.

Long-term climatological records are available for temperature, precipitation, and relative humidity. Statistics generated from these data are used for comparisons with the 2003 summaries.

Available temperature and relative humidity records begin in 1964. From 1964-1995, climatological statistics used in this report were based on the record of daily high and low values recorded at the 773-A instrument shelter. After 1995, these statistics were based on the continuous record of 15-minute data from the $2 \mathrm{~m}$ level of CLM.

Precipitation data are available from 1952. For the period 1952 through 1995, climatological statistics used in the report were based on the daily observations from the 773-A rain gauge. Summaries after 1995 were based on the automated 15minute records collected from the CLM rain gauge. In June 2003, apparent discrepancies were noted between monthly precipitation totals that had been generated using 773-A data from the relational database and totals published in other sources. Following a detailed investigation (Parker and Antonicelli, 2003), several problem areas were identified (most notably during the period 1965 through 1981) and a corrected electronic record was produced. 


\section{Climatology of the SRS for 2003}

\section{O verview}

Meteorological data summaries for 2003 indicate that weather conditions at SRS during the year were generally colder and wetter than long-term averages. Total annual precipitation of 61.2 inches was the third highest amount over all years in the available period of record. Higher totals were observed in 1964 (73.5 in) and 1971 (68.2 in). Furthermore, the annual average temperature of $62.2 \circ \mathrm{F}$ was the coldest of any year in the available historical record.

An annual average temperature of $62.2 \circ \mathrm{F}$ was also observed in 1996. An annual temperature of 62.5 of was observed in 2000 and 1981.

Cool and wet conditions were most pronounced in the spring and summer months. Unusually cold weather also occurred in January and December. A summary plot of 2003 daily observations of temperature, precipitation, relative humidity, and wind speed is given in Fig. 2.

These conditions are consistent with regional observations. Monthly climatological discussions published by the National Weather Service, Climate Prediction Center (CPC), indicate that the cool and wet weather observed throughout much of the Southeastern U. S. for January through April was due to the ongoing presence of El Nino conditions over the tropical Pacific Ocean. Hemispheric circulation patterns during El Nino feature an active subtropical jet over the southern U.S. with strong southwest to westerly winds aloft. This results in the frequent development of low pressure systems which move through the Southeast producing persistent cloudiness and ample rain.
Although the El Nino had largely dissipated by late spring, cool and wet conditions continued through the summer as a persistent upper-level low pressure trough developed over the Eastern U.S. Weak cool fronts were able to penetrate into the Southeast on several occasions. This upper low, combined with moist southerly flow from a Bermuda high located in a position off the Southeast U.S. coast, lead to enhanced development of afternoon and evening thunderstorm activity.

The upper level trough began to amplify during September and October. West to northwest flow on the southwestern flank of the trough enabled the frequent transport of cool, dry polar air masses into the area. Warmer weather returned in November as the upper trough position shifted westward and allowed an Atlantic subtropical ridge to build over the lower Southeast. The upper trough returned to a position along the east coast on several occasions in December, accompanied by a strong polar jet that was responsible for outbreaks of cold polar air from Canada (NOAA, 2003a and 2003b).

\section{Temperature}

Monthly and annual average and daily extreme temperatures for 2003 are summarized in Table 1(a). Similar statistics for a 30-year climatological reference period (1973-2002) are given in Table 2. A plot of observed daily high and low temperatures for 2003 is shown in Fig. 3. Plots of annual average temperature, monthly average daily high and low temperature, and days exceeding critical temperature thresholds ( $\angle 32 \mathrm{oF},>90 \mathrm{oF}$ ) for 2003 and the 30-year climatology are shown in figures 4, 5, and 6, respectively. 
The annual average temperature of $62.2 \mathrm{oF}$ was 2.4 degrees below the 30-year average and was the coldest annual average temperature for any year in the full period of record that dates to 1964 . Monthly averages for 2003 were below their respective climatological values for all months in the year except March and November. Furthermore, monthly averages for A pril, June, July, September, and December all ranked among the five coldest months on record. Temperatures above $90 \circ \mathrm{F}$ occurred on a total of only seventeen days during the year and only one-fourth of days in July and August. Temperature extremes ranged from $12.5 \mathrm{oF}$ on January 24 to 92.4 of on August 27. Daily low temperature records were set on January 18, 25, and 26, March 31, and September 29.

Monthly and annual total heating and cooling degree days (based on a reference temperature of $65 \circ$ F) are summarized in Table 1(c). The colder than average temperatures that occurred in 2003 resulted in a higher annual total heating degree days and a lower annual total cooling degree days than were observed over the last several years.

\section{Precipitation}

Annual, monthly, and daily total rainfall statistics for 2003 are summarized in Table 1(a). Monthly and annual rainfall totals for the 30-year climatology (1973-2002) are given in Table 3. Monthly and annual rainfall totals for the 12 plastic wedge gauges are summarized in Table 4. A plot of daily total rainfall (midnight to midnight) for the CLM site for 2003 is shown as Fig. 7. Annual and monthly rainfall for 2003 and the 30-year reference period are shown in figures 8 and 9, respectively.
Total precipitation at the CLM site, 61.18 inches, was nearly 12 inches greater than the 30-year average and resulted in the third wettest year over the 51 years of available data. Higher totals were observed in 1964 (73.5 in) and 1971 (68.2 in). Much of the annual surplus was due to rainfall occurring over the months March through July. Monthly totals for A pril and June were the highest and second highest totals on record for those months, respectively. The heavy rain that occurred on April 7 (3.5 in) was due to an active stationary front over the area and strong southwesterly winds al oft. The remnants of Tropical Storm Bill produced 2.36 in of rain on July 1 . Measurable precipitation $(>0.01$ inch) occurred on 119 days and rainfall greater than 0.5 inch occurred on 42 days. The total number of rain days was much higher in 2003 than for the previous several years.

\section{Atmospheric M oisture}

Monthly and annual average and daily extreme dew point temperature, wet bulb temperature, and relative humidity for 2003 are summarized in Table 1(b). This table also presents monthly and annual average daily maximum and minimum humidity. A plot of daily maximum and minimum humidity for 2003 is shown in Fig. 10. Plots of annual average humidity for 2003 and the 30-year climatology (19732002) and monthly average daily mi nimum humidity are shown in figures 11 and 12 , respectively.

Average relative humidity for 2003 was 70 percent with an average daily minimum of 48 percent and an average daily maximum of 87 percent. Days with relative humidity of 20 percent or less occurred in January, February, April, November, and December. The lowest relative humidity recorded during the year was 16 percent 
on November 13. A verage daily minimum relative humidity was higher than longterm averages throughout the spring and summer months.

Observed dew point temperatures ranged from 81.5 of on June 8 to 5.7 of on January 18. Wet bulb temperature ranged from 82.0 oF on June 8 to 14.5 oF on January 18.

\section{Heat Stress}

Restrictions on outdoor work due to excessive heat are based on values of the wet bulb globe temperature (WBGT) (WSRC, 1995). Hunter and Minyard (2000) provides a description of WBGT and the method used at SRS to calculate WBGT from standard meteorological measurements at CLM.

Monthly and annual maximum WBGT and statistics on heat stress category days for 2003 is summarized in Table 1(b). The highest WBGT for 2003 was 92.8 oF on June 30. Heat stress Category 5 days (WBGT > $90.0 \mathrm{oF}$ ) occurred on only 13 days during the summer of 2003. A total of 142 days reached at least Category 1 from April through November of the year. The number of occurrence days for each heat stress category in 2003 is considerably less than that for the previous several years.

\section{Wind}

Monthly average and extreme groundlevel wind speed (CLM 4 meter level) for 2003 is summarized in Table 1(c). Daily averaged wind speed for the year are plotted in Fig. 13. Figures 14(a) and 14(b) show wind rose plots depicting joint occurrence frequencies of the indicated wind speed category by wind direction sector at the 61 meter level of the eight area towers. Fig. 15 provides wind rose plots for the 4 levels of measurement at CLM.
Seasonal wind rose plots for 2003 for data from the $61 \mathrm{~m}$ level of CLM are shown in Fig. 16. Tables of the joint frequency data used to create the wind rose plots are given in A ppendix A.

Wind speeds were strongest on average during the winter and spring months. The highest instantaneous wind speed recorded at the $4 \mathrm{~m}$ level, $41.7 \mathrm{mph}$, was the result of a thunderstorm on May 3. A strong winter storm moving through the Southeast U. S. on February 23 produced the highest daily average wind speed of $11.2 \mathrm{mph}$.

Wind rose plots for the area towers show typical annual patterns for the 61 meter level. This pattern consists of higher frequencies of wind from the northeasterly sectors and southwest to westerly sectors. Due to the location of the $D$ area tower in the shallow valley formed by the Savannah River, wind direction is more frequently from the southeasterly and northwesterly sectors than for the other area towers. Wind roses for CLM also show typical variations in the frequency patterns by level, with progressively higher frequencies of southeasterly winds and lower frequencies of northeasterly and southwesterly winds nearer the ground. Weber (2003) provides a complete description of the wind climatology at the CLM site.

Seasonal wind rose plots for the 61 meter level at CLM are indicative of the typical seasonal patterns observed at SRS and generally reflect features of the larger scale weather patterns described above.

\section{Barometric Pressure}

Annual and monthly average and extreme barometric pressure is summarized in Table 1(c). Daily average barometric 
pressure is plotted in Fig. 17. The lowest daily average and 15-minute minimum barometric pressure, 992.1 and $984.4 \mathrm{mb}$, respectively, were associated with the winter storm of February 22-23. The highest daily average and 15-minute barometric pressure, 1020.2 and $1023.8 \mathrm{mb}$, respectively, were associated with strong polar high pressure that built over the Southeast U. S. December 20-21.

\section{Solar Radiation}

Annual and monthly averages and extremes of daily total solar radiation are summarized in Table 1(c). This table also provides estimated monthly and annual estimates of percent observed solar radiation relative to theoretical clear sky maxima as an indicator of relative cloudiness. The monthly theoretical values were estimated from tables published by Budyko (1974). Daily total solar radiation for 2003 is plotted in Fig. 18. The average daily values ranged from 236 langleys per day (ly/ day) in December to 506 ly/ day in June. The low value for daily average solar radiation in December is due to a low solar angle, as the percent of theoretical maximum for the month, $72 \%$, implies December was characterized by ample sunshine. Individual daily extremes ranged from $28 \mathrm{ly} /$ day on February 16 to $701 \mathrm{ly} /$ day on June 21 . 


\section{References}

Budyko, M. I., Climate and Life, Academic Press, New York, NY (1974).

Hunter, C. H., and C. O. Minyard, Estimating Wet Bulb Globe Temperature Using Standard Meteorological Measurements, American Meteorological Society $2^{\text {nd }}$ Symposium on Environmental Applications, January 9-14, 2000, Long Beach, CA (2000).

National Oceanic and Atmospheric Administration (NOAA), Climate Diagnostics Bulletin (January - December 2003), published electronically by month at www.cpc.ncep.noaa.gov, Climate Prediction Center, Camp Springs, MD(2003a).

NOAA, Daily Weather Maps, National Weather Service, Camp Springs, MD.(2003b)

Oliver, J. E. and R. W. Fairbridge, ed., The Encyclopedia of Climatology, Von Nostrand Reinhold, New York, NY (1987).

Parker, M. J. and R. P. Addis, M eteorological $M$ onitoring Program at the Savannah River Site, WSRC-TR-93-0106, Westinghouse Savannah River Company (1993).

Parker, M. J. and A. B. Antonicelli, Description of ATG's Rain History Table, WSRC Interoffice Memorandum SRT-NTS2003-00021, Westinghouse Savannah River Company (2003).
SRTC Nonproliferation Technologies Section, Quality Assurance of M eteorological Data, WSRC Procedure Manual 15.3, Meteorological Monitoring Procedures, NTSP T-113 (2002).

Weber, A. H., R. J. Kurzeja and R. L. Buckley, Wind Climate Analysis for SRTC's Central Climatology Site, WSRC-TR-200300141, Westinghouse Savannah River Company, Aiken, SC (2003).

Westinghouse Savannah River Company (WSRC), The SRS H eat Stress M anagement Program, Procedure Manual 4Q, Procedure 502, Revision 2 (1995). 
Table 1(a) - Means and Extremes of SRS Meteorological Data for 2003

\begin{tabular}{|c|c|c|c|c|c|c|c|c|c|c|c|c|c|c|c|c|c|c|c|c|c|}
\hline \multirow[b]{3}{*}{ 돋 } & \multicolumn{13}{|c|}{ Temperatures ( $\left.{ }^{\circ} \mathrm{F}\right)$} & \multicolumn{8}{|c|}{ Precipitation (in) } \\
\hline & \multicolumn{5}{|c|}{ Average } & \multicolumn{4}{|c|}{ Extremes } & \multicolumn{4}{|c|}{ Number of Days } & \multirow[b]{2}{*}{$\begin{array}{l}\bar{\pi} \\
\stackrel{0}{0}\end{array}$} & \multirow[b]{2}{*}{ 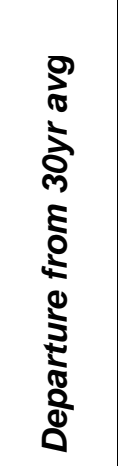 } & \multirow[b]{2}{*}{ 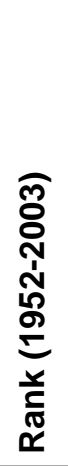 } & \multirow[b]{2}{*}{ 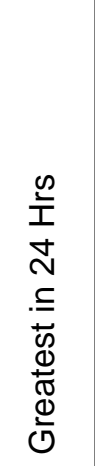 } & \multirow[b]{2}{*}{ 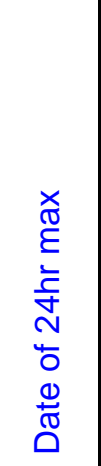 } & \multicolumn{3}{|c|}{ No. of Days } \\
\hline & 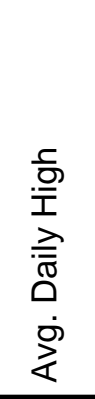 & 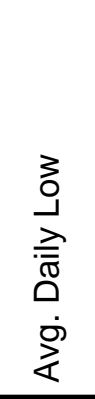 & 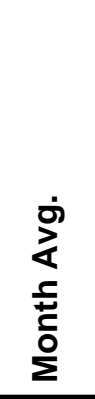 & 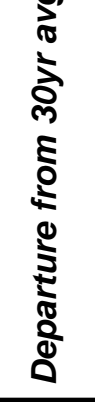 & 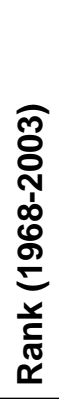 & 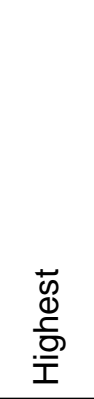 & 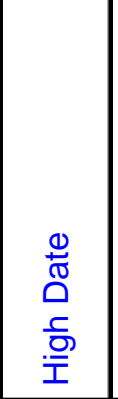 & $\begin{array}{l}\overleftarrow{n} \\
\$ \\
0 \\
0\end{array}$ & $\begin{array}{l}\stackrel{0}{ \pm} \\
0 \\
3 \\
0 \\
0\end{array}$ & 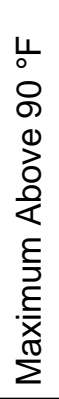 & 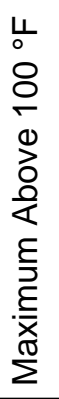 & 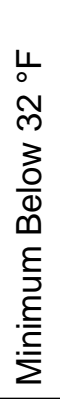 & 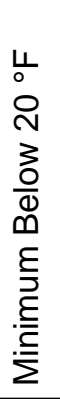 & & & & & & 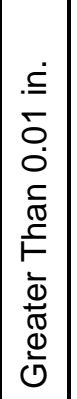 & 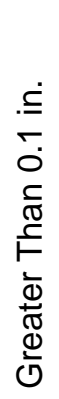 & 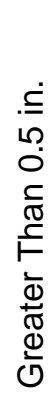 \\
\hline Jan & 53.9 & 32.6 & 42.0 & -4.1 & 6 & 70.7 & 9th & 12.5 & 24th & 0 & 0 & 15 & 3 & 1.73 & -2.69 & 6 & 0.86 & 30th & 5 & 4 & 1 \\
\hline Feb & 59.3 & 38.2 & 47.5 & -2.5 & 11 & 76.1 & 15th & 27.9 & 11th & 0 & 0 & 4 & 0 & 5.00 & +0.65 & 21 & 1.28 & 16th & 11 & 8 & 5 \\
\hline Mar & 68.6 & 48.8 & 57.6 & +0.3 & 15 & 79.9 & 29th & 29.9 & $31 \mathrm{st}$ & 0 & 0 & 2 & 0 & 7.09 & +2.17 & 10 & 1.85 & 20th & 14 & 9 & 5 \\
\hline Apr & 72.8 & 51.2 & 61.6 & -2.7 & 5 & 84.5 & 30th & 31.9 & $1 \mathrm{st}$ & 0 & 0 & 0 & 0 & 8.43 & +5.08 & 1 & 3.50 & 7th & 9 & 7 & 4 \\
\hline May & 81.3 & 62.3 & 70.6 & -1.4 & 11 & 89.6 & 10th & 49.9 & 13th & 0 & 0 & 0 & 0 & 5.57 & +1.93 & 7 & 1.38 & 19th & 10 & 8 & 4 \\
\hline Jun & 86.4 & 66.8 & 75.2 & -3.1 & 4 & 90.7 & 27th & 55.1 & 2nd & 1 & 0 & 0 & 0 & 10.99 & +6.10 & 2 & 2.27 & 18th & 13 & 11 & 8 \\
\hline Jul & 87.1 & 70.5 & 77.3 & -4.1 & 1 & 91.9 & 17th & 66.3 & 24th & 8 & 0 & 0 & 0 & 8.91 & +3.27 & 6 & 2.36 & 2nd & 16 & 12 & 6 \\
\hline Aug & 87.8 & 71.2 & 77.7 & -2.4 & 6 & 92.4 & 27th & 68.2 & 9th & 8 & 0 & 0 & 0 & 4.59 & -0.36 & 27 & 0.81 & $1 \mathrm{st}$ & 12 & 10 & 3 \\
\hline Sep & 82.2 & 63.9 & 71.9 & -3.1 & 5 & 89.3 & 4th & 47.5 & 30th & 0 & 0 & 0 & 0 & 2.70 & -1.32 & 13 & 0.76 & $1 \mathrm{st}$ & 10 & 7 & 1 \\
\hline Oct & 75.5 & 54.6 & 63.7 & -1.4 & 12 & 82.9 & $21 \mathrm{st}$ & 43.6 & 16th & 0 & 0 & 0 & 0 & 3.03 & -0.08 & 19 & 1.34 & 28th & 7 & 5 & 2 \\
\hline Nov & 71.5 & 48.0 & 58.2 & +1.5 & 12 & 84.0 & 5th & 26.2 & 30th & 0 & 0 & 2 & 0 & 1.21 & -1.69 & 12 & 0.57 & 19th & 7 & 3 & 1 \\
\hline Dec & 56.0 & 32.6 & 42.9 & -5.4 & 2 & 68.1 & 23rd & 21.0 & $21 s t$ & 0 & 0 & 16 & 0 & 1.93 & -1.41 & 13 & 0.65 & 10th & 5 & 4 & 2 \\
\hline Year & 73.6 & 53.5 & 62.2 & -2.4 & 1 & 92.4 & 27-Aug & 12.5 & 24-Jan & 17 & 0 & 39 & 3 & 61.18 & +11.64 & 3 & 3.50 & 7-Apr & 119 & 88 & 42 \\
\hline
\end{tabular}


Table 1(b) - Means and Extremes of SRS Meteorological Data for 2003

\begin{tabular}{|c|c|c|c|c|c|c|c|c|c|c|c|c|c|c|c|c|c|c|c|c|c|c|}
\hline \multirow[b]{3}{*}{$\begin{array}{l}\text { 三 } \\
\text { 을 }\end{array}$} & \multicolumn{5}{|c|}{ Dew Point Temperature $\left({ }^{\circ} \mathrm{F}\right)$} & \multicolumn{5}{|c|}{ Wet Bulb Temperature ( $\left.{ }^{\circ} \mathrm{F}\right)$} & \multicolumn{5}{|c|}{ Relative Humidity (\%) } & \multicolumn{7}{|c|}{ Wet Bulb Globe Temperature ( $\left.{ }^{\circ} \mathrm{F}\right)$} \\
\hline & \multirow[b]{2}{*}{ 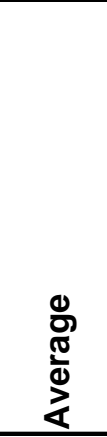 } & \multirow[b]{2}{*}{ 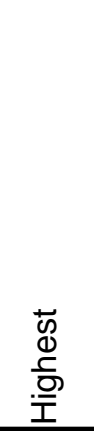 } & \multirow[b]{2}{*}{ 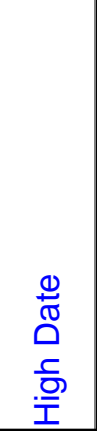 } & \multirow[b]{2}{*}{$\begin{array}{l}\bar{\omega} \\
ٍ \\
\vdots \\
0\end{array}$} & \multirow[b]{2}{*}{ 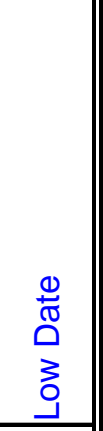 } & \multirow[b]{2}{*}{ 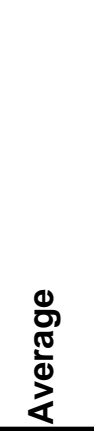 } & \multirow[b]{2}{*}{ 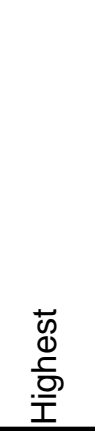 } & \multirow[b]{2}{*}{ 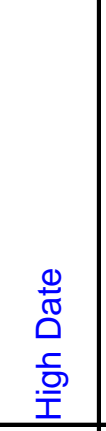 } & \multirow[b]{2}{*}{ 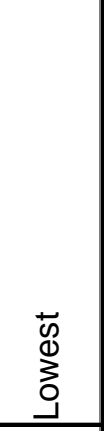 } & \multirow[b]{2}{*}{$\begin{array}{l}0 \\
\stackrel{0}{ \pm} \\
0 \\
3 \\
0 \\
\end{array}$} & \multirow{2}{*}{ 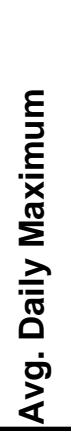 } & \multirow{2}{*}{ 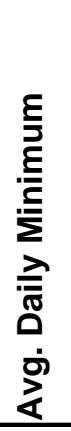 } & \multirow[b]{2}{*}{$\begin{array}{l}\underset{\lambda}{c} \\
\stackrel{\Sigma}{c} \\
\text { 을 }\end{array}$} & \multirow[b]{2}{*}{$\begin{array}{l}\text { ळ } \\
\$ \\
0 \\
\end{array}$} & \multirow[b]{2}{*}{\begin{tabular}{l}
0 \\
\multirow{0}{*}{} \\
0 \\
3 \\
0 \\
\end{tabular}} & \multirow[b]{2}{*}{ 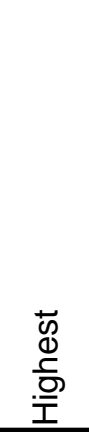 } & \multirow[b]{2}{*}{ 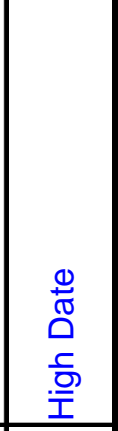 } & \multicolumn{5}{|c|}{ Number of Days } \\
\hline & & & & & & & & & & & & & & & & & & 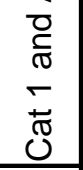 & 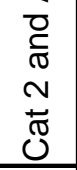 & 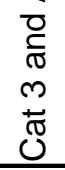 & 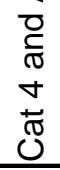 & $\begin{array}{l}0 \\
\mathbb{0} \\
0\end{array}$ \\
\hline Jan & 28.4 & 59.7 & $1 \mathrm{st}$ & 5.7 & 18th & 37.2 & 60.6 & $1 \mathrm{st}$ & 14.5 & 18th & 83 & 36 & 60 & 20 & 10th & 66.0 & $1 \mathrm{st}$ & 0 & 0 & 0 & 0 & 0 \\
\hline Feb & 36.5 & 64.7 & 22nd & 15.1 & 8th & 42.6 & 65.4 & 22nd & 27.2 & 11th & 91 & 45 & 70 & 20 & 13th & 71.0 & 15th & 0 & 0 & 0 & 0 & 0 \\
\hline Mar & 47.9 & 67.6 & 20th & 20.0 & $31 s t$ & 52.5 & 69.1 & 20th & 28.8 & $31 \mathrm{st}$ & 91 & 52 & 74 & 21 & 25th & 75.9 & 29th & 0 & 0 & 0 & 0 & 0 \\
\hline Apr & 49.8 & 68.8 & 7th & 28.0 & $1 \mathrm{st}$ & 55.2 & 69.0 & 7th & 30.7 & $1 \mathrm{st}$ & 91 & 45 & 70 & 20 & 13th & 80.2 & 29th & 3 & 0 & 0 & 0 & 0 \\
\hline May & 61.7 & 72.2 & 18th & 41.8 & 12th & 65.2 & 74.7 & 10th & 53.4 & 30th & 91 & 51 & 75 & 26 & 12th & 85.5 & 8th & 22 & 10 & 2 & 0 & 0 \\
\hline Jun & 65.6 & 81.5 & 8th & 48.3 & 2nd & 69.1 & 82.0 & 8th & 52.6 & 2nd & 93 & 51 & 75 & 33 & $1 \mathrm{st}$ & 92.8 & 30th & 29 & 24 & 18 & 7 & 2 \\
\hline Jul & 69.5 & 78.6 & 27th & 63.1 & 14th & 72.1 & 79.8 & 27 th & 65.3 & 25th & 90 & 60 & 78 & 49 & 9th & 90.5 & 17th & 29 & 27 & 26 & 16 & 5 \\
\hline Aug & 68.4 & 76.6 & 25th & 57.0 & 5 th & 71.5 & 78.6 & 16th & 61.8 & 5 th & 87 & 55 & 74 & 49 & 23rd & 91.4 & 24th & 31 & 30 & 27 & 16 & 6 \\
\hline Sep & 59.8 & 76.3 & $3 r d$ & 37.5 & 29th & 64.6 & 78.3 & 3rd & 43.2 & 30th & 83 & 47 & 68 & 28 & 28th & 88.8 & 2nd & 21 & 10 & 6 & 2 & 0 \\
\hline Oct & 51.8 & 72.7 & 14th & 33.2 & $3 r d$ & 57.1 & 74.9 & 14th & 39.3 & $3 r d$ & 83 & 47 & 68 & 25 & 22nd & 81.1 & 14th & 3 & 0 & 0 & 0 & 0 \\
\hline Nov & 46.0 & 70.4 & 5th & 16.1 & 14th & 52.0 & 74.2 & 5 th & 24.0 & 30th & 80 & 45 & 66 & 16 & 13th & 85.3 & 4th & 4 & 2 & 1 & 0 & 0 \\
\hline Dec & 30.8 & 58.0 & 10th & 13.5 & $21 \mathrm{st}$ & 37.9 & 59.4 & 10th & 19.0 & $21 \mathrm{st}$ & 83 & 42 & 65 & 19 & 2nd & 65.9 & $23 r d$ & 0 & 0 & 0 & 0 & 0 \\
\hline Year & 51.4 & 81.5 & 8-Jun & 5.7 & 18-Jan & 56.4 & 82.0 & 8-Jun & 14.5 & 18-Jan & 87 & 48 & 70 & 16 & 13-Nov & 92.8 & 30-Jun & 142 & 103 & 80 & 41 & 13 \\
\hline
\end{tabular}


Table 1(c) - Means and Extremes of SRS Meteorological Data for 2003 (cont'd)

\begin{tabular}{|c|c|c|c|c|c|c|c|c|c|c|c|c|c|c|c|c|c|c|c|c|c|}
\hline \multirow[b]{2}{*}{$\begin{array}{l}\text { 壱 } \\
\text { 을 }\end{array}$} & \multicolumn{4}{|c|}{ Wind Speed (mph) } & \multicolumn{5}{|c|}{ Barometric Pressure (mb) } & \multicolumn{6}{|c|}{ Solar Radiation (ly) } & \multicolumn{6}{|c|}{ Degree Days } \\
\hline & 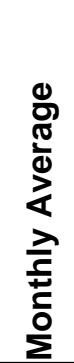 & 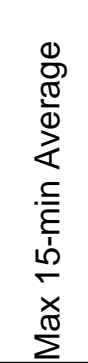 & 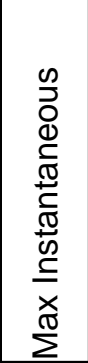 & 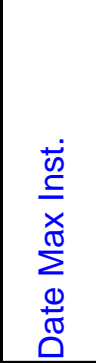 & $\begin{array}{l}\frac{0}{\mathbb{2}} \\
\frac{\pi}{0} \\
\frac{0}{\alpha}\end{array}$ & $\begin{array}{l}\bar{\phi} \\
0 \\
0 \\
0\end{array}$ & $\begin{array}{l}\stackrel{0}{ \pm} \\
0 \\
3 \\
0 \\
\end{array}$ & 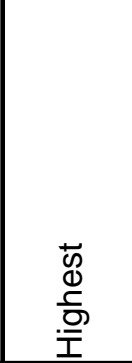 & 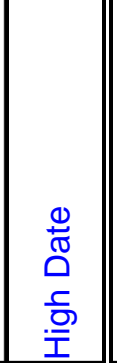 & 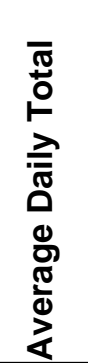 & 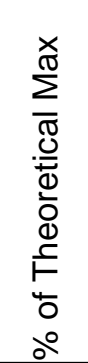 & 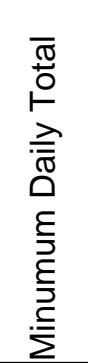 & $\frac{0}{\pi}$ & 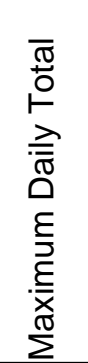 & $\begin{array}{l}0 \\
\stackrel{ \pm}{0} \\
0\end{array}$ & 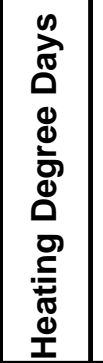 & 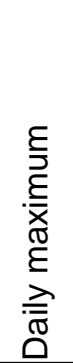 & $\frac{0}{\tilde{0}}$ & 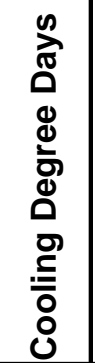 & 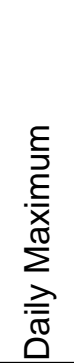 & $\frac{0}{\widetilde{D}}$ \\
\hline Jan & 4.9 & 16.5 & 32.6 & 23rd & 1008.8 & 994.1 & 9th & 1022.0 & 24th & 256 & 0.71 & 67 & 30th & 368 & 27 th & 710 & 42 & 24th & 0 & 0 & \\
\hline Feb & 4.7 & 17.2 & 38.5 & $23 r d$ & 1006.6 & 984.4 & 23rd & 1016.4 & 19th & 259 & 0.58 & 28 & 16th & 463 & $23 r d$ & 489 & 32 & 17th & 0 & 0 & \\
\hline Mar & 4.2 & 15.0 & 36.9 & 20th & 1003.6 & 991.0 & 18th & 1016.1 & $31 \mathrm{st}$ & 306 & 0.54 & 35 & 1st & 597 & $31 \mathrm{st}$ & 237 & 22 & $31 \mathrm{st}$ & 7 & 4 & 29th \\
\hline Apr & 4.2 & 13.1 & 25.5 & 22nd & 1005.4 & 991.7 & 26th & 1018.9 & $1 \mathrm{st}$ & 430 & 0.63 & 49 & 7th & 661 & 23rd & 127 & 20 & 10th & 24 & 6 & 30th \\
\hline May & 4.3 & 17.5 & 41.7 & 3rd & $\mid$\begin{tabular}{||l}
1003.1 \\
\end{tabular} & 990.2 & $31 \mathrm{st}$ & 1013.5 & 20th & 436 & 0.58 & 98 & 22nd & 678 & 12th & 5 & 3 & 14th & 176 & 14 & 9th \\
\hline Jun & 3.6 & 14.3 & 35.8 & 13th & $\mid 1004.2$ & 990.5 & $1 \mathrm{st}$ & \begin{tabular}{|l|l}
1013.8 \\
\end{tabular} & 30th & 506 & 0.64 & 188 & 7th & 701 & $21 \mathrm{st}$ & 0 & 0 & & 307 & 14 & 27th \\
\hline Jul & 3.7 & 15.2 & 38.4 & 7th & $\mid$\begin{tabular}{||c|c} 
\\
1007.0
\end{tabular} & 998.8 & 2nd & 1014.8 & 26th & 466 & 0.61 & 128 & $1 \mathrm{st}$ & 630 & 9th & 0 & 0 & & 385 & 17 & 9th \\
\hline Aug & 3.1 & 11.7 & 27.4 & 2nd & 1006.8 & 999.0 & 8th & 1016.4 & 14th & 438 & 0.62 & 261 & 12th & 622 & 14th & 0 & 0 & & 396 & 17 & 27th \\
\hline Sep & 3.7 & 16.2 & 31.5 & 4th & 1006.3 & 997.0 & 18th & 1015.0 & 30th & 426 & 0.70 & 116 & 7th & 560 & 17th & 8 & 5 & 30th & 216 & 13 & 4th \\
\hline Oct & 3.4 & 15.9 & 31.4 & 15th & 1005.9 & 992.7 & 14th & 1016.5 & $31 \mathrm{st}$ & 333 & 0.67 & 35 & 28th & 506 & $3 r d$ & 76 & 8 & 16th & 35 & 6 & 22nd \\
\hline Nov & 4.0 & 19.6 & 35.4 & 19th & 1009.6 & 992.2 & 19th & \begin{tabular}{|l}
1021.3 \\
\end{tabular} & 9th & 271 & 0.68 & 78 & 7th & 392 & 2nd & 236 & 26 & 30th & 32 & 9 & 6th \\
\hline Dec & 4.1 & 16.8 & 35.4 & 10th & 1009.9 & 990.5 & 10th & \begin{tabular}{|l}
1023.8 \\
\end{tabular} & $21 \mathrm{st}$ & 236 & 0.72 & 34 & 4th & 326 & 2nd & 684 & 32 & $21 \mathrm{st}$ & 0 & 0 & \\
\hline Year & 4.0 & 19.6 & 41.7 & 3-May & 1006.4 & 984.4 & 23-Feb & 1023.8 & 21-Dec & 364 & 0.64 & 28 & 16-Feb & 701 & 21-Jun & \begin{tabular}{|l|l|}
2573 \\
\end{tabular} & 42 & 24-Jan & 1578 & 17 & 27-Aug \\
\hline
\end{tabular}


Table 2. Monthly and Annual Average and Extreme Temperatures, 1973-2003

\begin{tabular}{|c|c|c|c|c|c|c|c|c|c|c|c|c|c|}
\hline Year & Jan & Feb & Mar & Apr & May & Jun & Jul & Aug & Sep & Oct & Nov & Dec & Annual \\
\hline 1973 & 46.1 & 45.9 & 60.7 & 61.9 & 70.5 & 77.7 & 79.1 & 74.5 & 70.5 & 62.4 & 59.0 & 50.3 & 63.2 \\
\hline 1974 & 59.6 & 50.8 & 62.2 & 66.2 & 75.3 & 77.5 & 81.5 & 80.9 & 75.3 & 64.5 & 56.6 & 49.0 & 66.6 \\
\hline 1975 & 51.4 & 53.2 & 55.8 & 63.9 & 75.6 & 79.1 & 79.7 & 82.4 & 75.7 & 68.7 & 59.3 & 48.5 & 66.1 \\
\hline 1976 & 44.2 & 55.7 & 61.5 & 64.8 & 68.9 & 75.6 & 80.4 & 78.0 & 73.1 & 60.1 & 48.7 & 44.8 & 63.0 \\
\hline 1977 & 35.3 & 47.1 & 60.0 & 66.9 & 73.3 & 80.6 & 83.6 & 80.6 & 77.9 & 62.1 & 58.2 & 46.7 & 64.4 \\
\hline 1978 & 39.3 & 41.3 & 54.2 & 65.7 & 70.9 & 79.7 & 82.1 & 81.2 & 77.1 & 65.6 & 60.7 & 49.6 & 64.0 \\
\hline 1979 & 42.1 & 44.6 & 57.5 & 64.5 & 71.3 & 75.1 & 79.6 & 80.5 & 73.4 & 64.8 & 57.4 & 47.4 & 63.2 \\
\hline 1980 & 45.9 & 44.3 & 52.6 & 63.5 & 71.2 & 78.3 & 83.8 & 82.5 & 79.2 & 62.7 & 52.8 & 46.0 & 63.6 \\
\hline 1981 & 40.4 & 48.5 & 53.0 & 67.0 & 68.6 & 81.3 & 81.3 & 76.3 & 74.0 & 62.1 & 54.4 & 43.2 & 62.5 \\
\hline 1982 & 43.0 & 50.0 & 58.9 & 62.4 & 75.7 & 78.8 & 80.9 & 80.1 & 75.0 & 66.2 & 58.7 & 54.8 & 65.4 \\
\hline 1983 & 43.3 & 48.0 & 55.3 & 59.4 & 66.8 & 76.7 & 84.3 & 83.9 & 74.8 & 67.2 & 56.4 & 45.8 & 63.5 \\
\hline 1984 & 45.0 & 51.7 & 56.5 & 62.6 & 71.9 & 80.1 & 80.1 & 80.8 & 74.0 & 73.4 & 53.4 & 56.9 & 65.5 \\
\hline 1985 & 42.9 & 49.5 & 60.2 & 67.5 & 74.5 & 80.8 & 81.1 & 79.7 & 75.7 & 70.8 & 65.5 & 45.4 & 66.1 \\
\hline 1986 & 45.4 & 54.6 & 57.9 & 66.4 & 74.4 & 82.7 & 86.9 & 80.1 & 78.4 & 67.1 & 61.3 & 49.3 & 67.0 \\
\hline 1987 & 46.2 & 48.6 & 56.5 & 62.3 & 74.5 & 79.9 & 82.8 & 83.8 & 76.6 & 60.7 & 59.1 & 52.9 & 65.3 \\
\hline 1988 & 42.3 & 47.8 & 56.8 & 64.2 & 70.4 & 76.8 & 81.6 & 81.4 & 75.4 & 61.2 & 58.0 & 49.1 & 63.8 \\
\hline 1989 & 52.2 & 52.0 & 58.3 & 64.2 & 70.6 & 79.8 & 81.4 & 80.9 & 75.3 & 67.3 & 52.4 & 44.2 & 64.9 \\
\hline 1990 & 54.9 & 57.5 & 60.0 & 64.0 & 72.9 & 80.5 & 83.7 & 83.8 & 79.0 & 69.4 & 59.9 & 54.6 & 68.4 \\
\hline 1991 & 47.9 & 54.1 & 60.3 & 69.2 & 76.9 & 79.5 & 83.6 & 81.2 & 77.4 & 68.1 & 55.4 & 54.0 & 67.3 \\
\hline 1992 & 49.5 & 54.1 & 57.2 & 65.0 & 71.2 & 78.9 & 83.7 & 80.7 & 76.9 & 65.0 & 57.1 & 48.0 & 65.6 \\
\hline 1993 & 51.7 & 47.8 & 53.2 & 58.9 & 69.7 & 78.2 & 83.6 & 80.0 & 75.2 & 62.8 & 55.2 & 43.6 & 63.3 \\
\hline 1994 & 41.5 & 50.1 & 60.2 & 68.0 & 71.2 & 82.3 & 81.8 & 81.2 & 77.4 & 67.2 & 62.3 & 53.3 & 66.4 \\
\hline 1995 & 45.5 & 49.9 & 58.6 & 65.9 & 73.5 & 75.0 & 79.9 & 79.0 & 71.8 & 65.9 & 50.8 & 43.8 & 63.3 \\
\hline 1996 & 44.6 & 50.1 & 50.6 & 61.6 & 72.9 & 76.5 & 79.3 & 76.0 & 72.7 & 62.1 & 51.6 & 48.8 & 62.2 \\
\hline 1997 & 48.2 & 52.9 & 63.3 & 61.2 & 68.5 & 74.0 & 80.2 & 79.0 & 75.0 & 64.1 & 51.6 & 47.0 & 63.8 \\
\hline 1998 & 49.7 & 51.1 & 53.6 & 62.7 & 74.6 & 82.1 & 82.6 & 80.3 & 75.8 & 66.9 & 60.5 & 53.6 & 66.1 \\
\hline 1999 & 51.9 & 51.6 & 53.4 & 67.2 & 69.7 & 76.6 & 80.7 & 82.9 & 73.8 & 64.3 & 58.1 & 48.6 & 64.9 \\
\hline 2000 & 44.4 & 50.2 & 58.5 & 60.7 & 75.1 & 78.0 & 79.9 & 77.6 & 71.7 & 62.5 & 53.1 & 38.2 & 62.5 \\
\hline 2001 & 43.8 & 52.4 & 53.0 & 63.9 & 71.3 & 75.3 & 77.7 & 78.8 & 71.2 & 62.2 & 60.0 & 52.4 & 63.5 \\
\hline 2002 & 47.3 & 48.0 & 57.6 & 68.1 & 70.2 & 77.5 & 80.5 & 78.4 & 75.4 & 66.7 & 51.7 & 44.5 & 63.8 \\
\hline 2003 & 42.0 & 47.5 & 57.6 & 61.6 & 70.6 & 75.2 & 77.3 & 77.7 & 71.9 & 63.7 & 58.2 & 42.9 & 62.2 \\
\hline
\end{tabular}

\begin{tabular}{|c|c|c|c|c|c|c|c|c|c|c|c|c|c|}
\hline Avg & 46.0 & 50.0 & 57.3 & 64.2 & 72.0 & 78.4 & 81.4 & 80.1 & 75.1 & 65.1 & 56.7 & 48.3 & 64.6 \\
\hline Lowest Mon & 35.3 & 41.3 & 50.6 & 58.9 & 66.8 & 74.0 & 77.3 & 74.5 & 70.5 & 60.1 & 48.7 & 38.2 & 62.2 \\
\hline Yr Lowest & 1977 & 1978 & 1971 & 1993 & 1983 & 1997 & 1967 & 1973 & 1973 & 1976 & 1976 & 2000 & 1996 \\
\hline Rec Low & -3 & 10 & 11 & 29 & 38 & 48 & 56 & 56 & 41 & 28 & 18 & 5 & -3 \\
\hline Yr Rec & 1985 & 1996 & 1980 & 1983 & 1989 & 1984 & 1963 & 1986 & 1967 & 1976 & 1970 & 1962 & 1985 \\
\hline Highest Mon & 59.6 & 57.5 & 63.3 & 69.2 & 76.9 & 82.7 & 86.9 & 83.9 & 79.2 & 73.4 & 65.5 & 56.9 & 68.4 \\
\hline Yr Highest & 1974 & 1990 & 1997 & 1991 & 1991 & 1986 & 1986 & 1983 & 1980 & 1984 & 1985 & 1971 & 1990 \\
\hline Rec High & 86 & 86 & 90 & 99 & 102 & 105 & 107 & 107 & 104 & 96 & 89 & 82 & 107 \\
\hline Yr Rec & 1975 & 1989 & 1974 & 1986 & 1963 & 1985 & 1986 & 1983 & 1990 & 1986 & 1987 & 1984 & 1986 \\
\hline
\end{tabular}


Table 3. Monthly and Annual Rainfall, 1973-2003

\begin{tabular}{|c|c|c|c|c|c|c|c|c|c|c|c|c|c|}
\hline Year & Jan & Feb & Mar & Apr & May & Jun & Jul & Aug & Sep & Oct & Nov & Dec & Annual \\
\hline 1973 & 5.50 & 4.47 & 6.67 & 4.55 & 4.91 & 12.97 & 6.86 & 3.90 & 4.38 & 1.72 & 0.98 & 3.99 & 60.90 \\
\hline 1974 & 2.42 & 6.66 & 3.03 & 3.05 & 3.35 & 2.80 & 4.44 & 6.77 & 3.32 & 0.09 & 1.99 & 4.11 & 42.03 \\
\hline 1975 & 4.98 & 6.64 & 5.92 & 4.42 & 5.15 & 3.83 & 8.55 & 3.83 & 5.18 & 1.74 & 3.41 & 2.03 & 55.68 \\
\hline 1976 & 4.18 & 1.08 & 3.83 & 2.50 & 10.90 & 4.35 & 1.95 & 1.64 & 5.48 & 4.92 & 4.19 & 5.08 & 50.10 \\
\hline 1977 & 3.72 & 1.62 & 6.86 & 1.27 & 1.79 & 2.47 & 3.42 & 7.30 & 5.50 & 4.27 & 1.63 & 3.86 & 43.71 \\
\hline 1978 & 10.02 & 1.31 & 3.06 & 3.53 & 3.64 & 3.42 & 4.11 & 5.10 & 4.06 & 0.06 & 3.54 & 1.87 & 43.72 \\
\hline 1979 & 3.59 & 7.74 & 3.09 & 6.49 & 8.94 & 1.54 & 7.85 & 2.12 & 6.13 & 1.35 & 3.95 & 2.17 & 54.96 \\
\hline 1980 & 5.12 & 3.48 & 10.96 & 1.69 & 3.49 & 2.99 & 0.90 & 2.03 & 5.86 & 2.14 & 2.50 & 1.91 & 43.07 \\
\hline 1981 & 0.89 & 5.02 & 4.72 & 2.07 & 6.90 & 4.29 & 3.96 & 5.79 & 0.54 & 2.81 & 1.00 & 9.55 & 47.54 \\
\hline 1982 & 3.94 & 4.46 & 2.51 & 5.68 & 2.73 & 4.28 & 11.49 & 5.02 & 4.62 & 3.87 & 2.41 & 4.85 & 55.86 \\
\hline 1983 & 3.75 & 7.22 & 6.62 & 5.77 & 1.67 & 6.57 & 4.85 & 6.32 & 3.56 & 1.92 & 5.39 & 4.15 & 57.79 \\
\hline 1984 & 3.51 & 7.09 & 6.05 & 8.00 & 9.79 & 2.54 & 7.28 & 5.52 & 0.60 & 0.31 & 0.90 & 1.38 & 52.97 \\
\hline 1985 & 3.01 & 6.92 & 1.31 & 0.84 & 1.70 & 4.62 & 8.10 & 4.38 & 0.49 & 6.34 & 6.36 & 2.48 & 46.55 \\
\hline 1986 & 1.46 & 3.58 & 4.08 & 1.45 & 3.84 & 3.03 & 2.96 & 10.90 & 1.54 & 4.19 & 5.82 & 5.83 & 48.68 \\
\hline 1987 & 7.39 & 7.55 & 4.97 & 0.70 & 3.57 & 5.64 & 4.87 & 4.93 & 3.56 & 0.29 & 2.74 & 1.42 & 47.63 \\
\hline 1988 & 4.15 & 3.19 & 2.91 & 4.78 & 2.85 & 7.12 & 1.78 & 6.80 & 4.40 & 3.39 & 2.17 & 2.91 & 46.45 \\
\hline 1989 & 1.42 & 3.59 & 5.52 & 4.89 & 2.60 & 6.67 & 11.46 & 3.27 & 4.87 & 3.36 & 3.00 & 4.41 & 55.06 \\
\hline 1990 & 3.07 & 2.38 & 2.37 & 1.21 & 2.95 & 0.89 & 7.31 & 8.07 & 0.62 & 19.62 & 1.41 & 1.57 & 51.47 \\
\hline 1991 & 7.03 & 1.84 & 7.89 & 4.73 & 3.06 & 2.17 & 7.89 & 9.26 & 4.40 & 0.99 & 1.55 & 3.32 & 54.13 \\
\hline 1992 & 4.45 & 3.89 & 2.98 & 2.40 & 1.34 & 6.27 & 3.69 & 4.83 & 6.38 & 3.11 & 7.78 & 2.86 & 49.98 \\
\hline 1993 & 7.45 & 3.62 & 8.37 & 1.74 & 1.43 & 3.27 & 3.12 & 2.23 & 7.29 & 0.99 & 1.87 & 1.81 & 43.19 \\
\hline 1994 & 4.80 & 3.91 & 6.42 & 1.05 & 1.45 & 5.08 & 7.47 & 3.47 & 0.99 & 10.01 & 3.05 & 4.62 & 52.32 \\
\hline 1995 & 6.96 & 7.97 & 0.92 & 1.28 & 1.77 & 8.15 & 5.71 & 6.92 & 5.75 & 2.64 & 2.38 & 4.47 & 54.92 \\
\hline 1996 & 3.65 & 2.43 & 6.64 & 2.40 & 2.96 & 3.04 & 5.57 & 6.91 & 3.67 & 2.16 & 2.32 & 3.20 & 44.95 \\
\hline 1997 & 4.20 & 5.45 & 2.69 & 4.38 & 2.38 & 6.90 & 7.09 & 2.01 & 4.89 & 4.08 & 5.51 & 9.09 & 58.67 \\
\hline 1998 & 7.73 & 8.90 & 6.69 & 7.35 & 4.05 & 4.65 & 5.27 & 2.88 & 4.81 & 0.78 & 0.82 & 1.80 & 55.73 \\
\hline 1999 & 5.31 & 2.29 & 3.44 & 1.95 & 1.26 & 7.52 & 4.91 & 3.14 & 4.46 & 2.57 & 1.50 & 1.21 & 39.56 \\
\hline 2000 & 5.77 & 0.73 & 3.95 & 1.34 & 1.36 & 4.74 & 2.47 & 4.49 & 7.70 & 0.02 & 3.50 & 1.53 & 37.60 \\
\hline 2001 & 3.11 & 2.68 & 7.21 & 1.28 & 3.85 & 6.49 & 4.79 & 3.55 & 3.33 & 0.50 & 1.03 & 0.54 & 38.36 \\
\hline 2002 & 2.85 & 2.13 & 3.86 & 2.58 & 1.69 & 2.30 & 5.95 & 5.47 & 3.45 & 3.19 & 4.00 & 3.58 & 41.05 \\
\hline 2003 & 1.73 & 5.00 & 7.09 & 8.43 & 5.57 & 10.99 & 8.91 & 4.59 & 2.70 & 3.03 & 1.21 & 1.93 & 61.18 \\
\hline Avg & 4.42 & 4.35 & 4.92 & 3.35 & 3.64 & 4.89 & 5.64 & 4.95 & 4.02 & 3.11 & 2.90 & 3.34 & 49.54 \\
\hline Min & 0.89 & 0.73 & 0.92 & 0.60 & 1.31 & 0.89 & 0.90 & 1.04 & 0.49 & 0.00 & 0.21 & 0.46 & 28.82 \\
\hline Yr Min & 1981 & 2000 & 1995 & 1972 & 1965 & 1990 & 1980 & 1963 & 1985 & 1963 & 1958 & 1955 & 1954 \\
\hline Max & 10.02 & 8.90 & 10.96 & 8.43 & 10.90 & 12.97 & 13.71 & 12.34 & 8.71 & 19.62 & 7.78 & 9.55 & 73.47 \\
\hline Yr Max & 1978 & 1998 & 1980 & 2003 & 1976 & 1973 & 1971 & 1964 & 1959 & 1990 & 1992 & 1981 & 1964 \\
\hline
\end{tabular}


Table 4 - SRS Rainfall (in inches) for 2003, Manual Gauges

\begin{tabular}{|c|c|c|c|c|c|c|c|c|c|c|c|c|}
\hline Month & 交 & 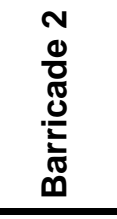 & 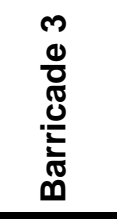 & 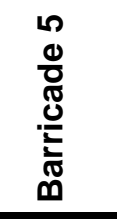 & 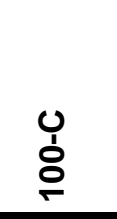 & 영 & 녕 & $\begin{array}{l}\text { I } \\
\text { ঠ্் }\end{array}$ & 농 & 형 & 옹 & $\frac{0}{\frac{v}{0}}$ \\
\hline Jan & 3.19 & 3.24 & 1.24 & 2.19 & 1.83 & 2.07 & 1.62 & 2.00 & 3.12 & 1.86 & 1.70 & 2.32 \\
\hline Feb & 5.00 & 5.80 & 4.86 & 4.18 & 5.20 & 5.31 & 5.97 & 6.63 & 5.45 & 4.85 & 5.72 & 5.03 \\
\hline Mar & 11.32 & 9.30 & 8.40 & 9.32 & 6.90 & 8.07 & 8.10 & 8.30 & 8.65 & 7.87 & 7.38 & 8.65 \\
\hline Apr & 9.93 & 11.59 & 10.26 & 7.37 & 8.38 & 8.64 & 9.67 & 9.52 & 7.33 & 6.27 & 7.53 & 9.19 \\
\hline May & 7.22 & 9.93 & 6.02 & 6.49 & 5.49 & 6.81 & 6.60 & 5.77 & 7.96 & 5.27 & 5.87 & 7.17 \\
\hline Jun & 9.01 & 10.37 & 9.60 & 10.26 & 10.88 & 9.18 & 7.28 & 8.60 & 11.72 & 10.30 & 8.88 & 9.47 \\
\hline Jul & 4.73 & 6.46 & 11.49 & 12.34 & 9.18 & 10.14 & 5.86 & 7.71 & 10.11 & 10.83 & 8.67 & 5.94 \\
\hline Aug & 3.95 & 6.03 & 6.68 & 5.30 & 4.26 & 3.96 & 3.09 & 3.77 & 4.93 & 8.64 & 9.54 & 5.16 \\
\hline Sep & 4.42 & 4.41 & 1.43 & 2.43 & 2.27 & 2.63 & 2.32 & 2.37 & 1.62 & 1.88 & 1.13 & 4.29 \\
\hline Oct & 3.35 & 3.29 & 3.73 & 3.39 & 2.91 & 3.39 & 3.10 & 3.09 & 3.62 & 2.80 & 2.77 & 3.31 \\
\hline Nov & 1.45 & 1.39 & 2.00 & 0.66 & 1.52 & 1.19 & 1.30 & 1.18 & 1.45 & 0.95 & 1.19 & 1.52 \\
\hline Dec & 1.61 & 2.11 & 2.37 & 2.70 & 2.15 & 2.26 & 2.27 & 2.03 & 1.70 & 1.59 & 1.63 & 1.92 \\
\hline Annual & 65.18 & 73.92 & 68.08 & 66.63 & 60.97 & 63.65 & 57.18 & 60.97 & 67.66 & 63.11 & 62.01 & 63.97 \\
\hline
\end{tabular}


Fig. 1 SRS Meteorological Monitoring Network

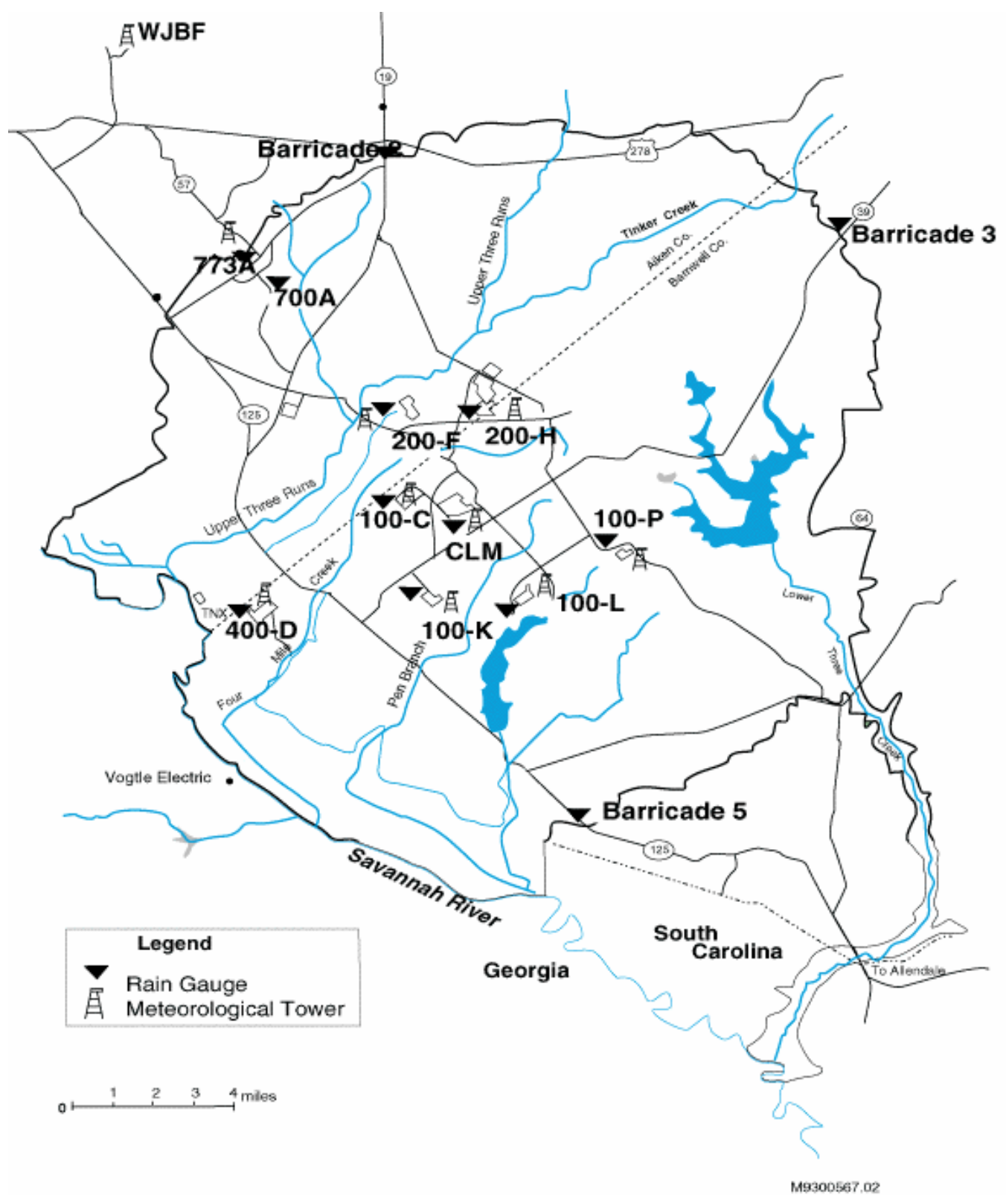


Fig. 2 - Summary of D aily D ata for 2003
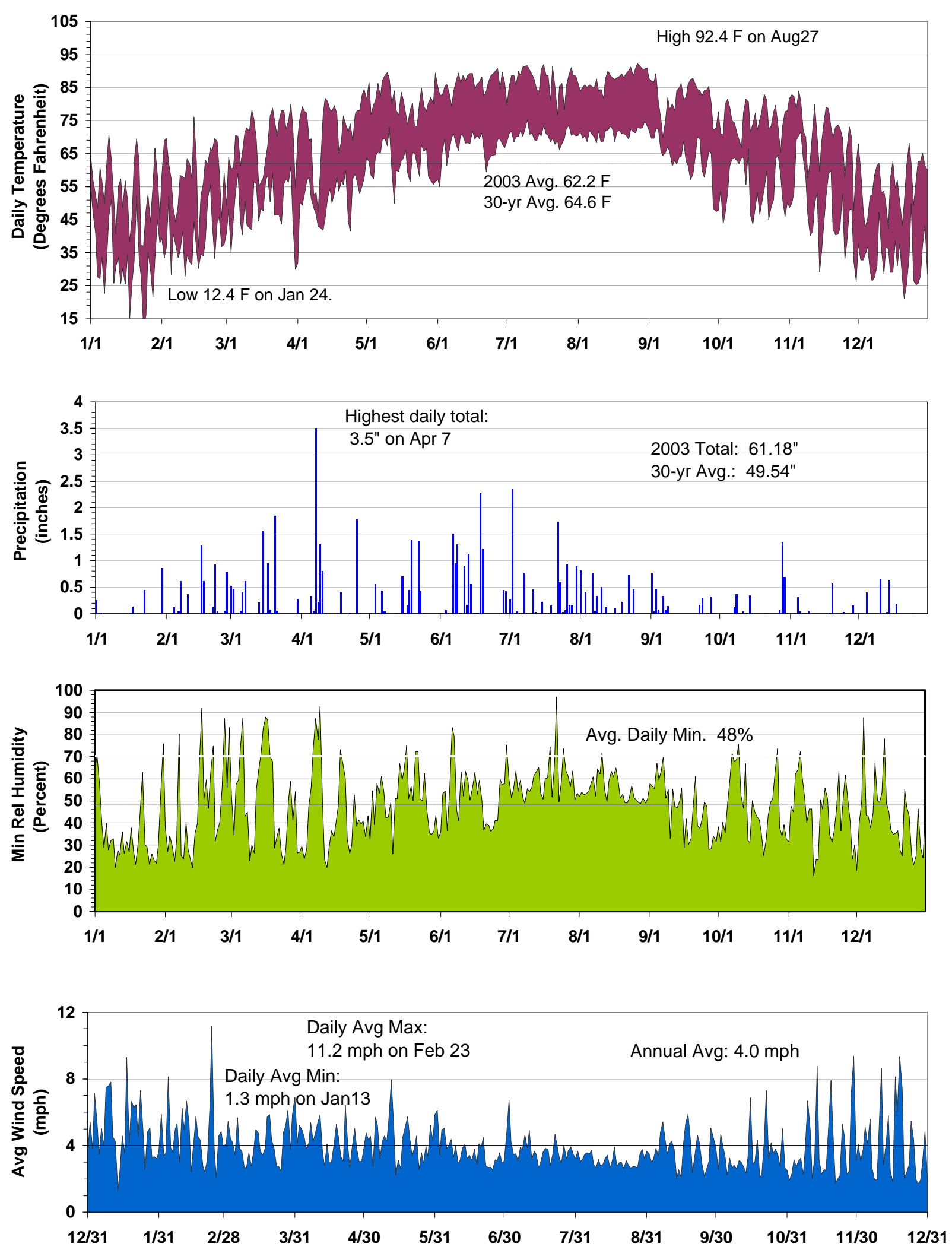
Fig. 3 - Daily High and Low Temperatures for 2003

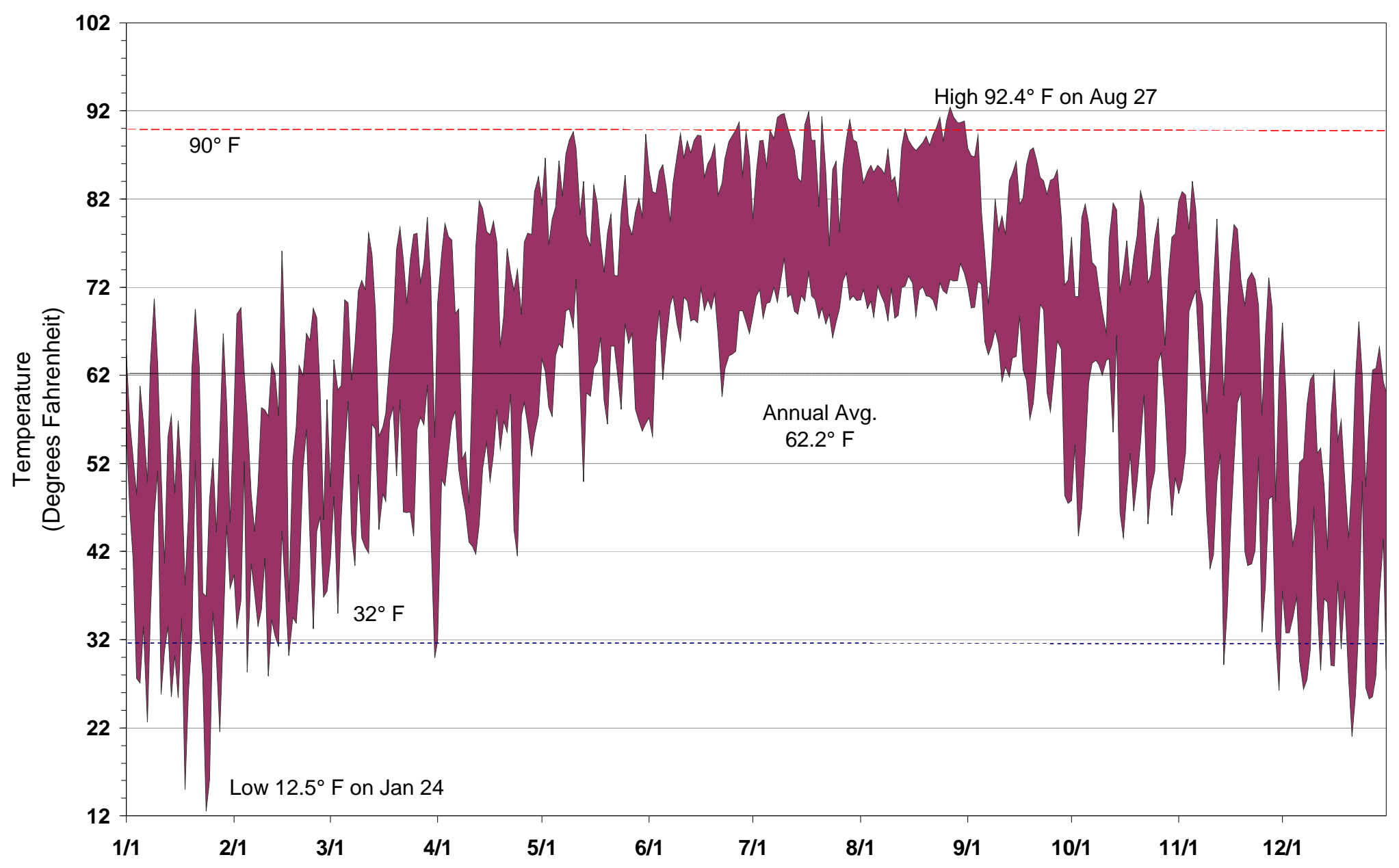


Fig. 4 - SRS Annual Average Temperature 1973-2003

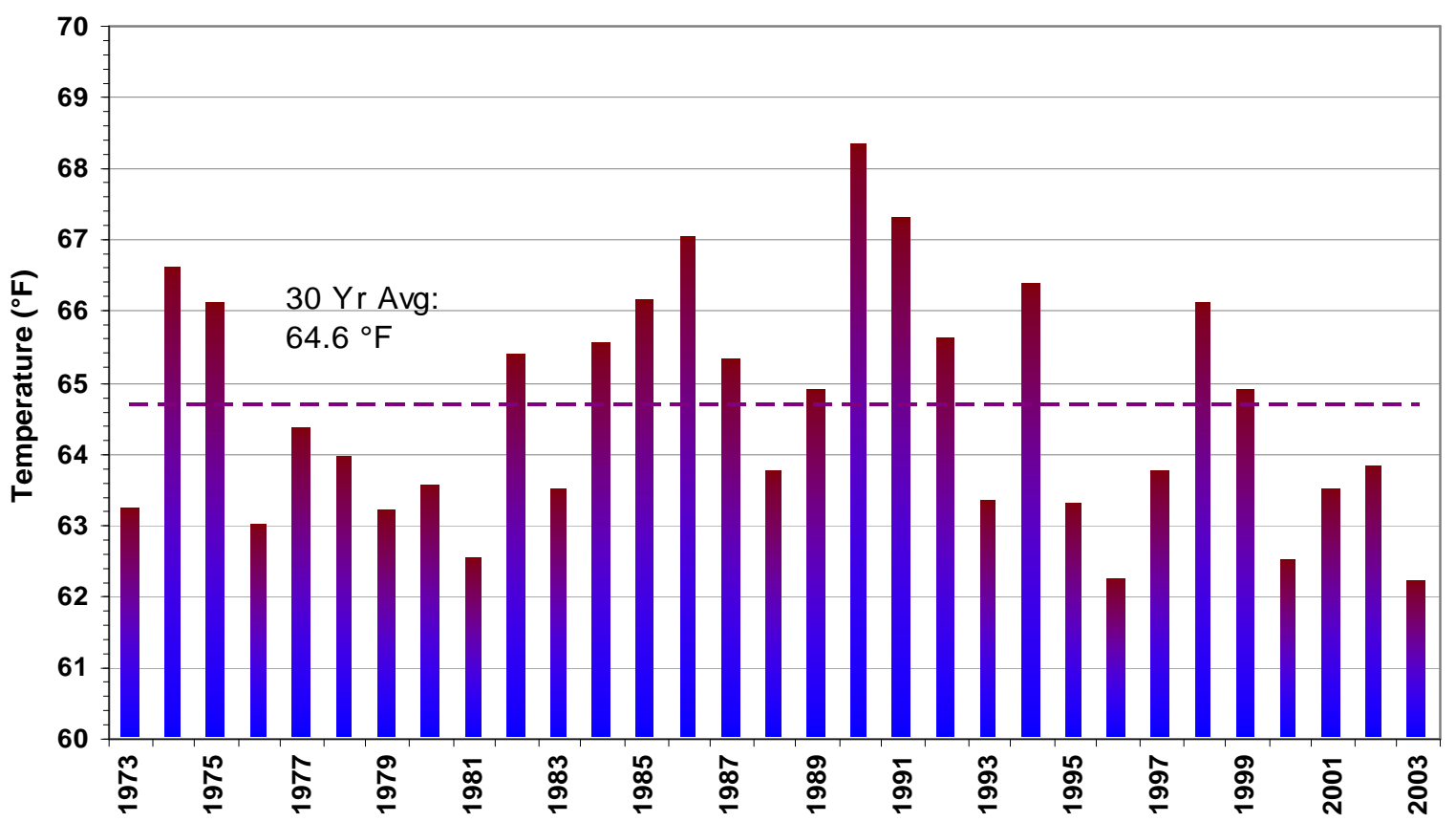

Fig. 5 - SRS Monthly Average Temperature

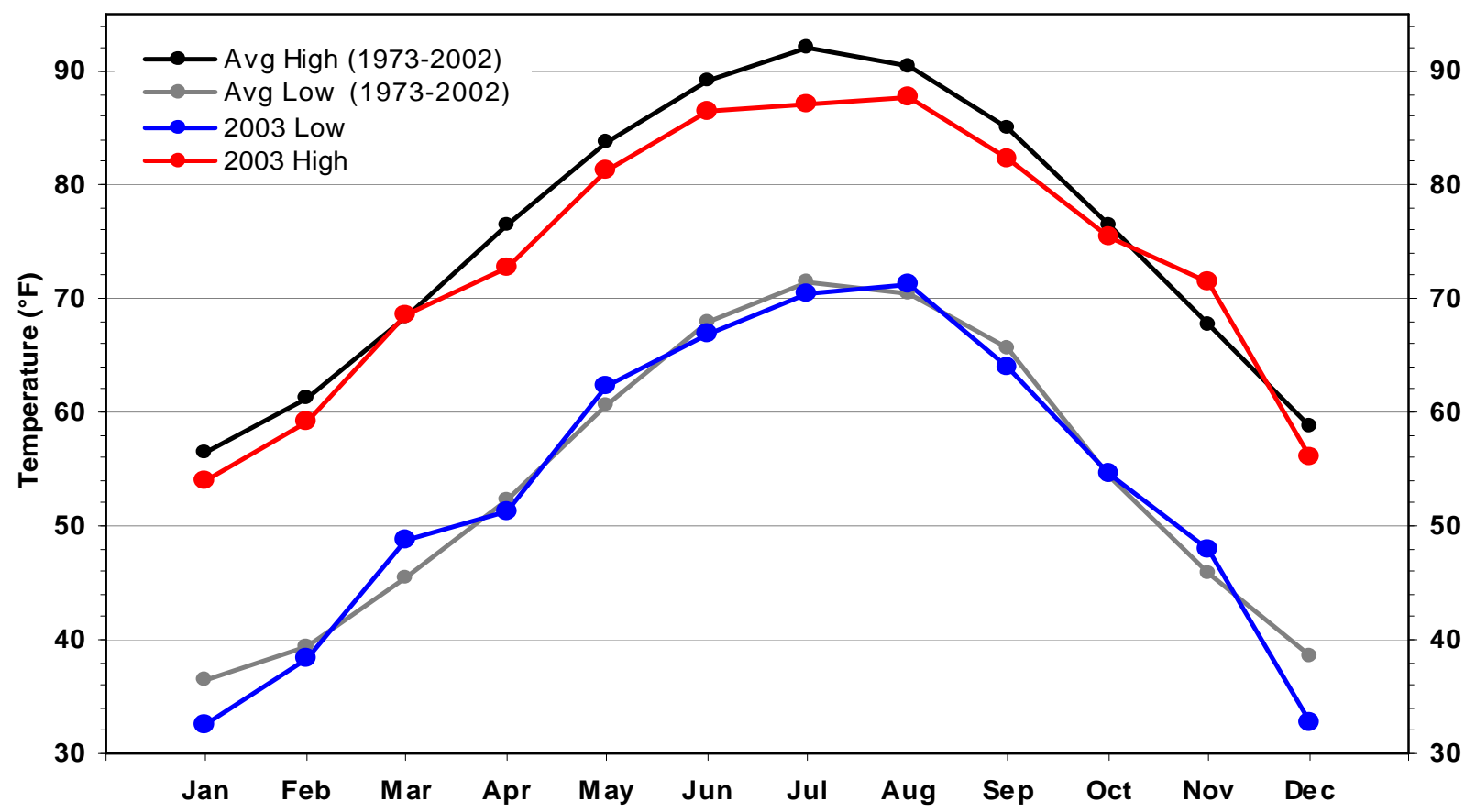


Fig. 6 - Number of Freezing (<32 F) and Sweltering ( $>90$ F) Days

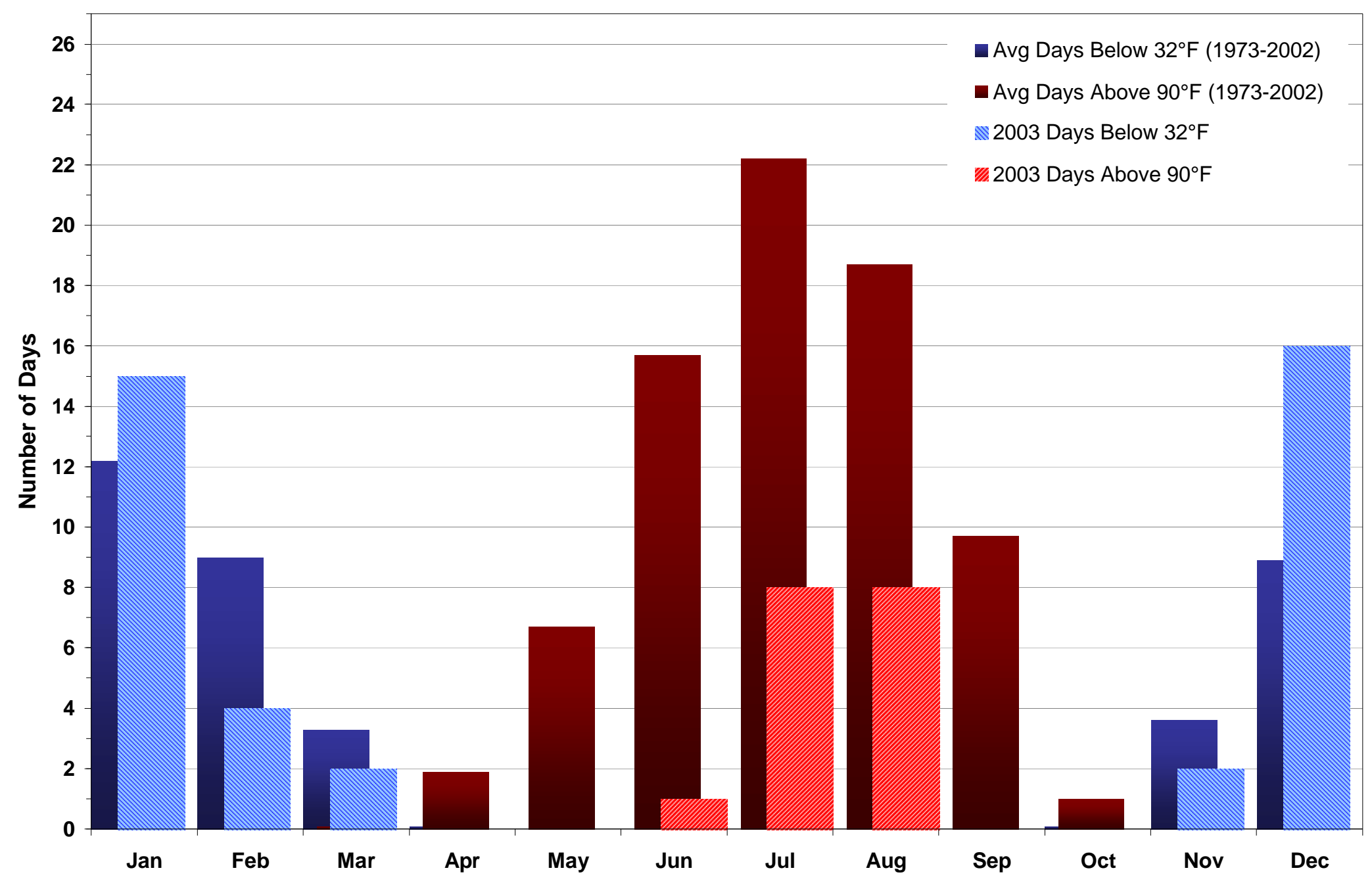




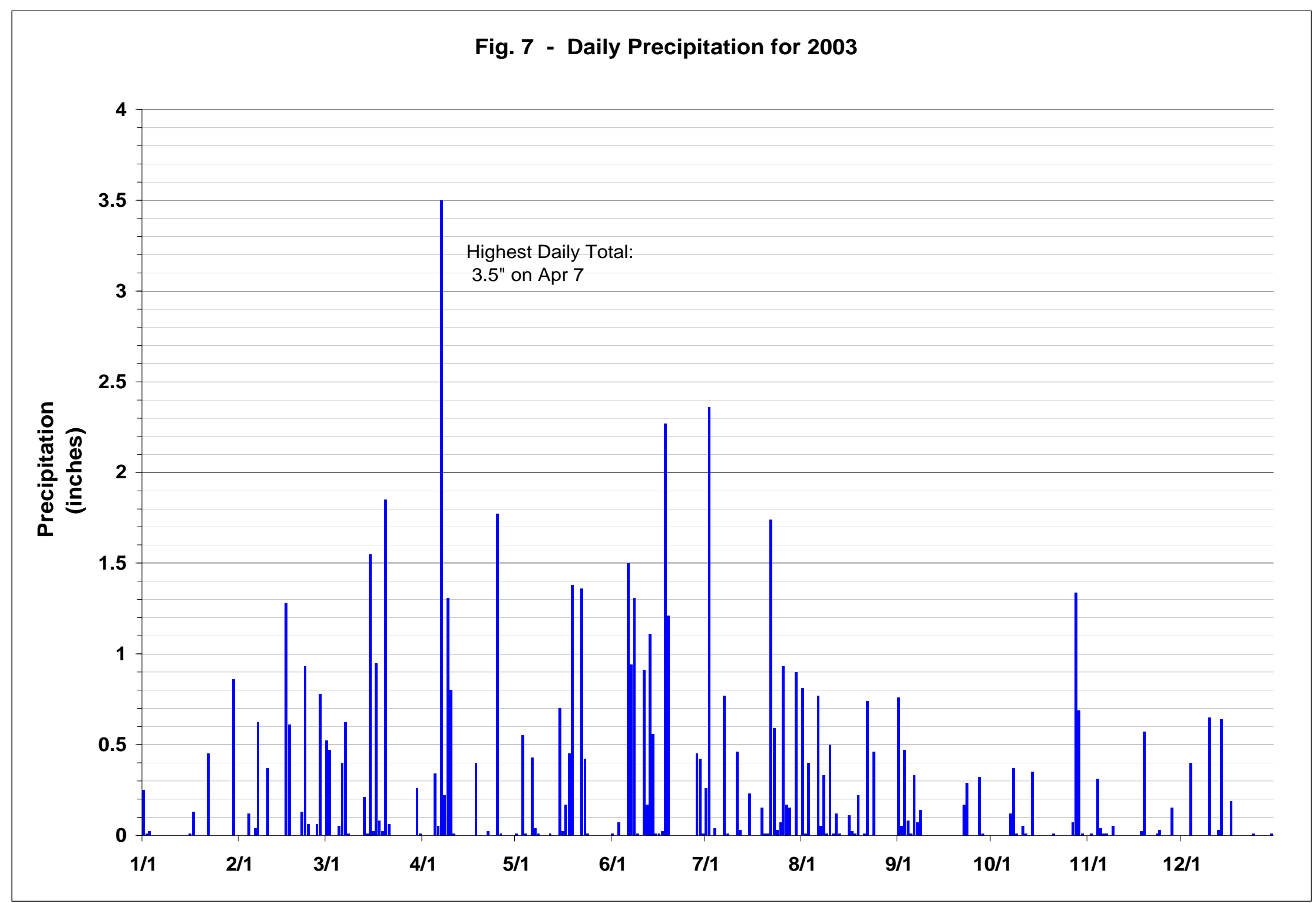


Fig. 8 - SRS Annual Precipitation 1973-2003

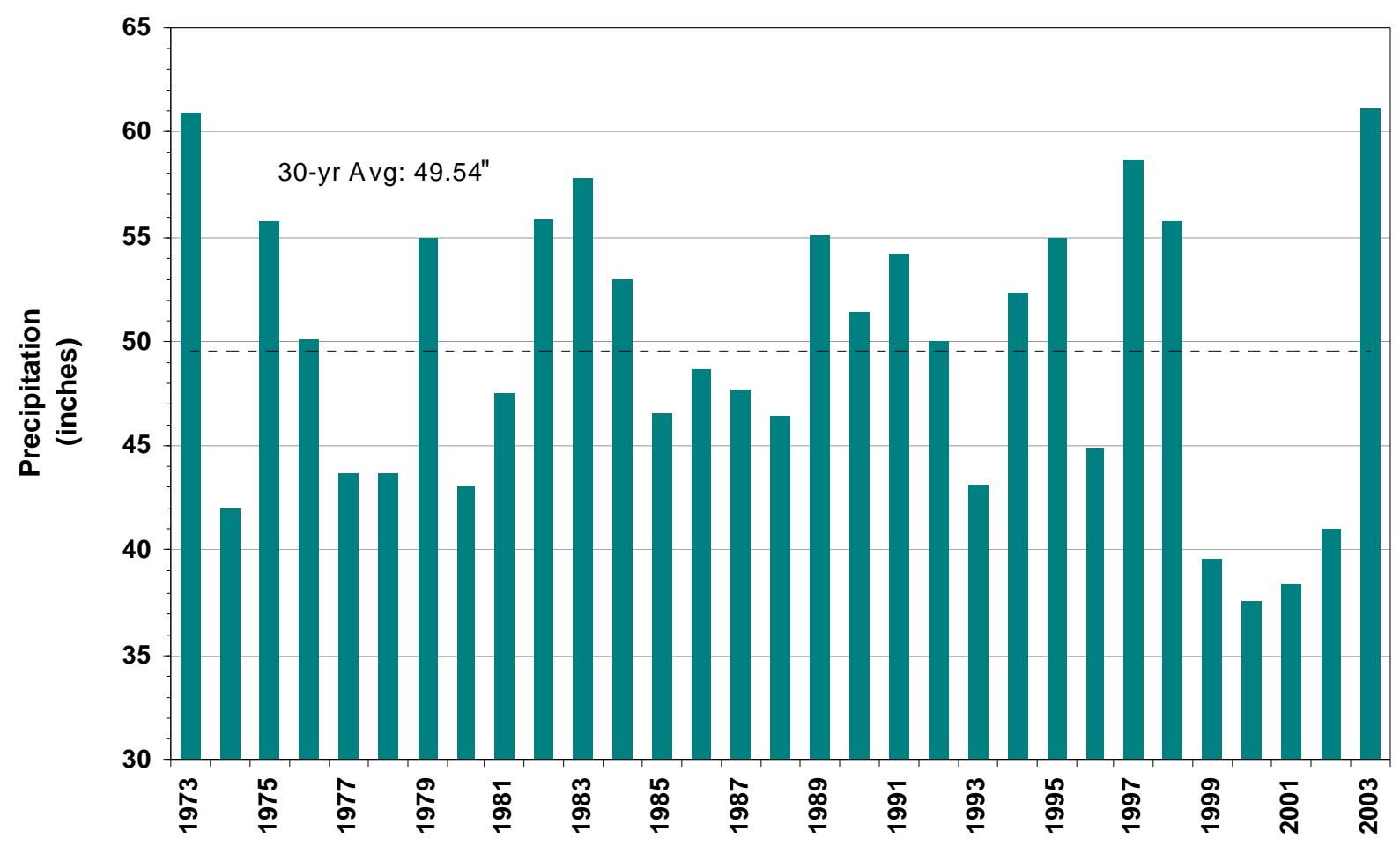

Fig. 9 - SRS Monthly Precipitation

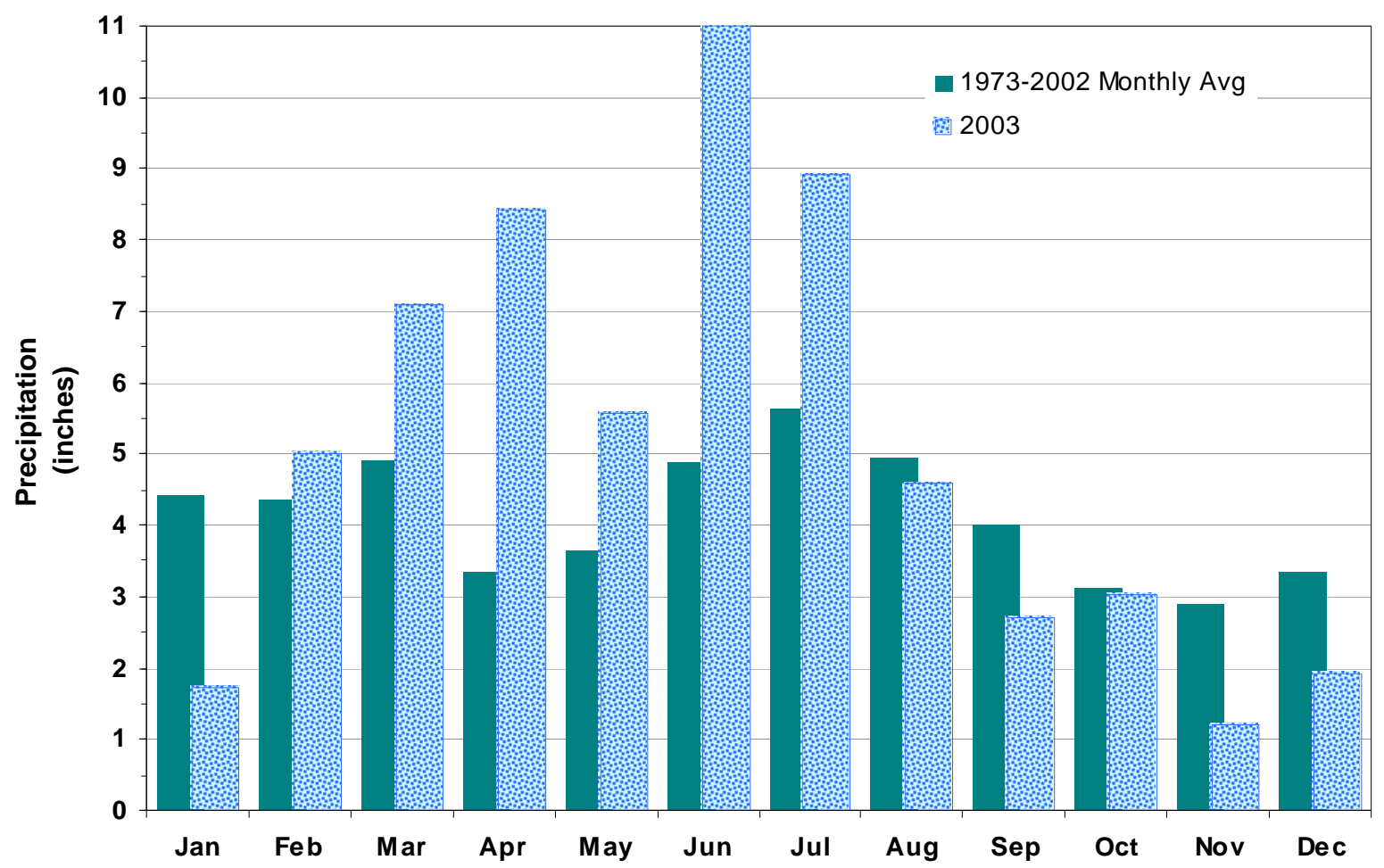


Fig. 10 - Daily High and Low Humidity for 2003

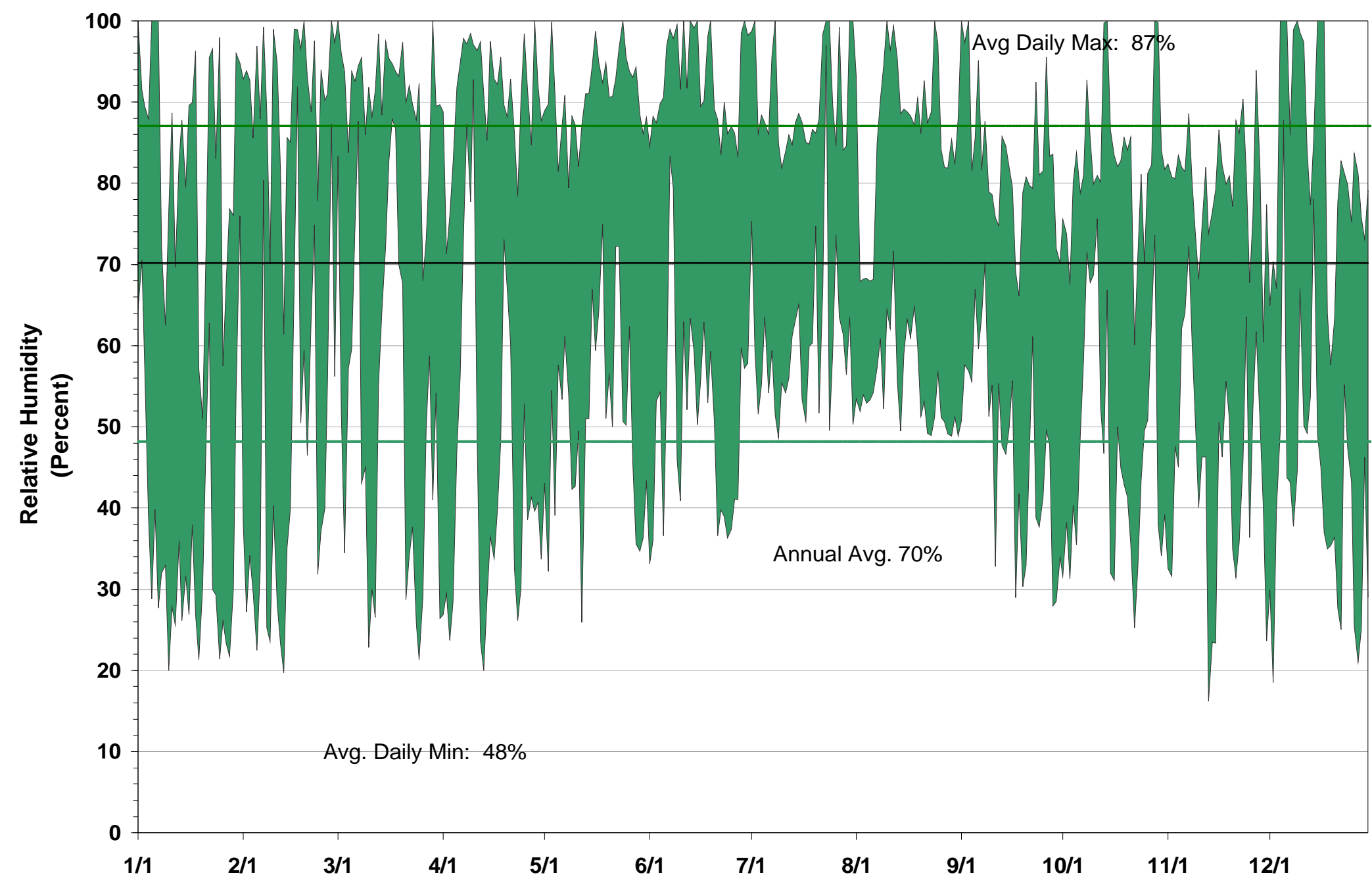


Fig. 11 - SRS Annual Average Humidity 1973-2003

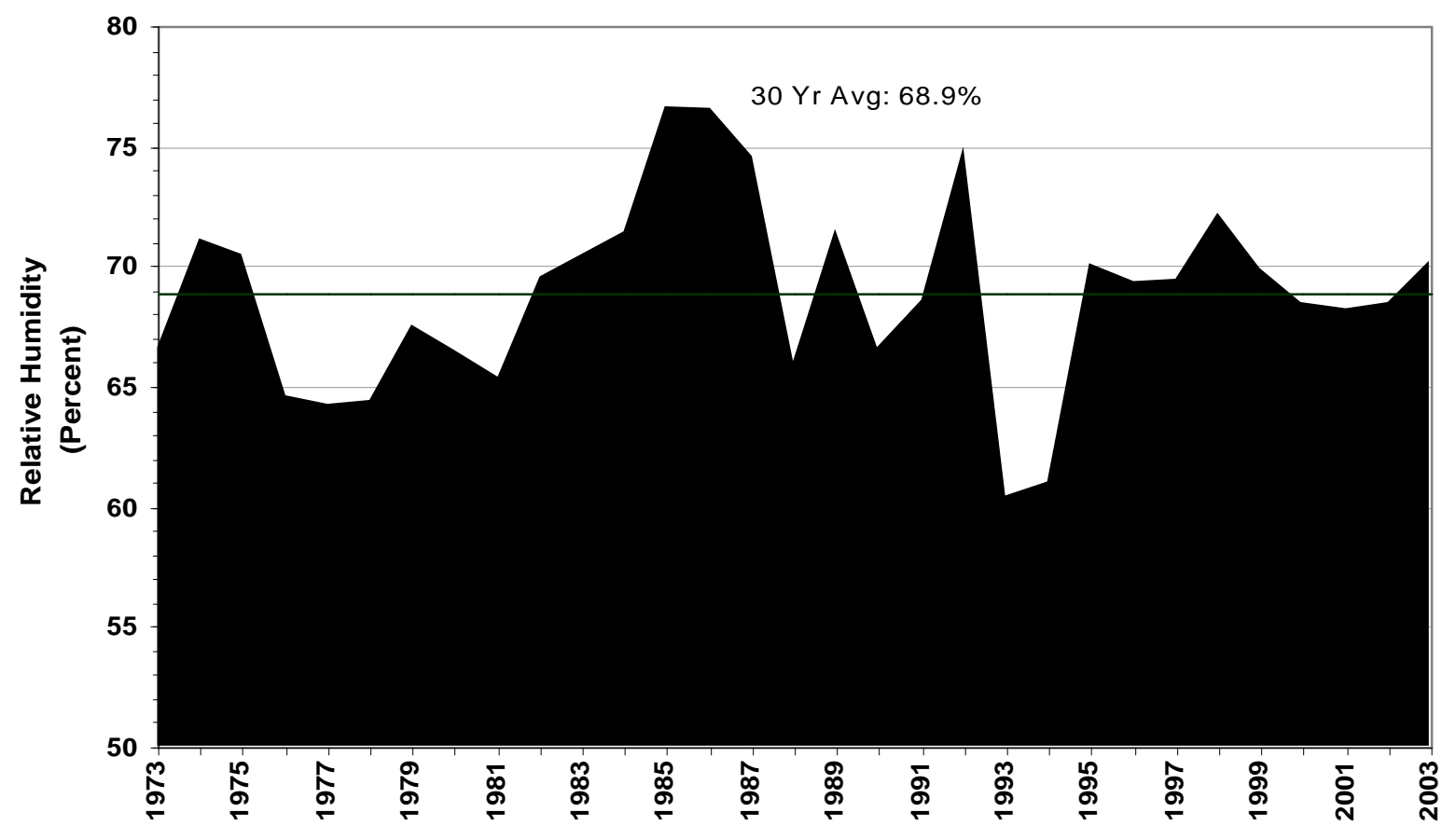

Fig. 12 - SRS Monthly Average Minimum Humidity

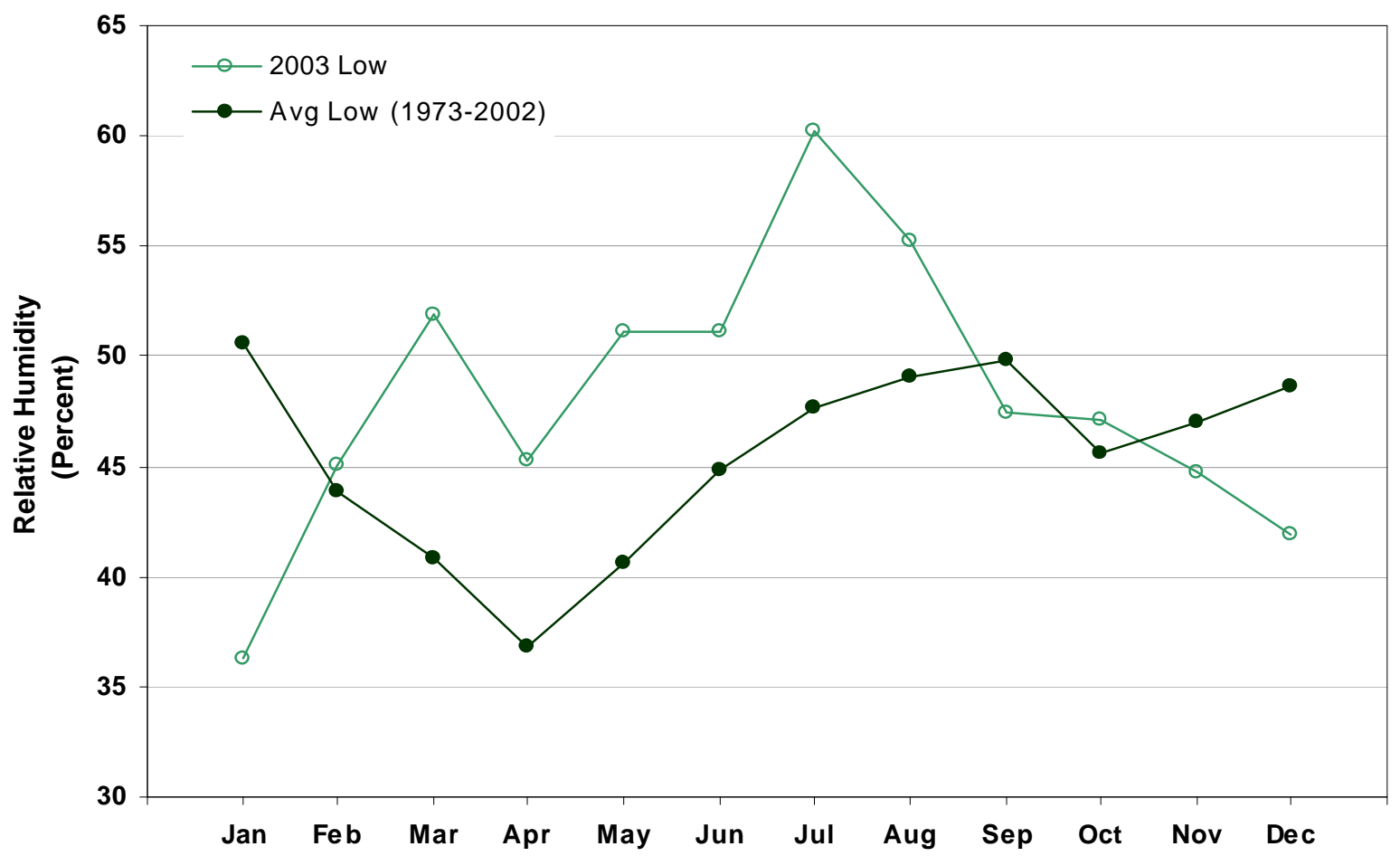


Fig. 13 - Daily Average Wind Speed for 2003

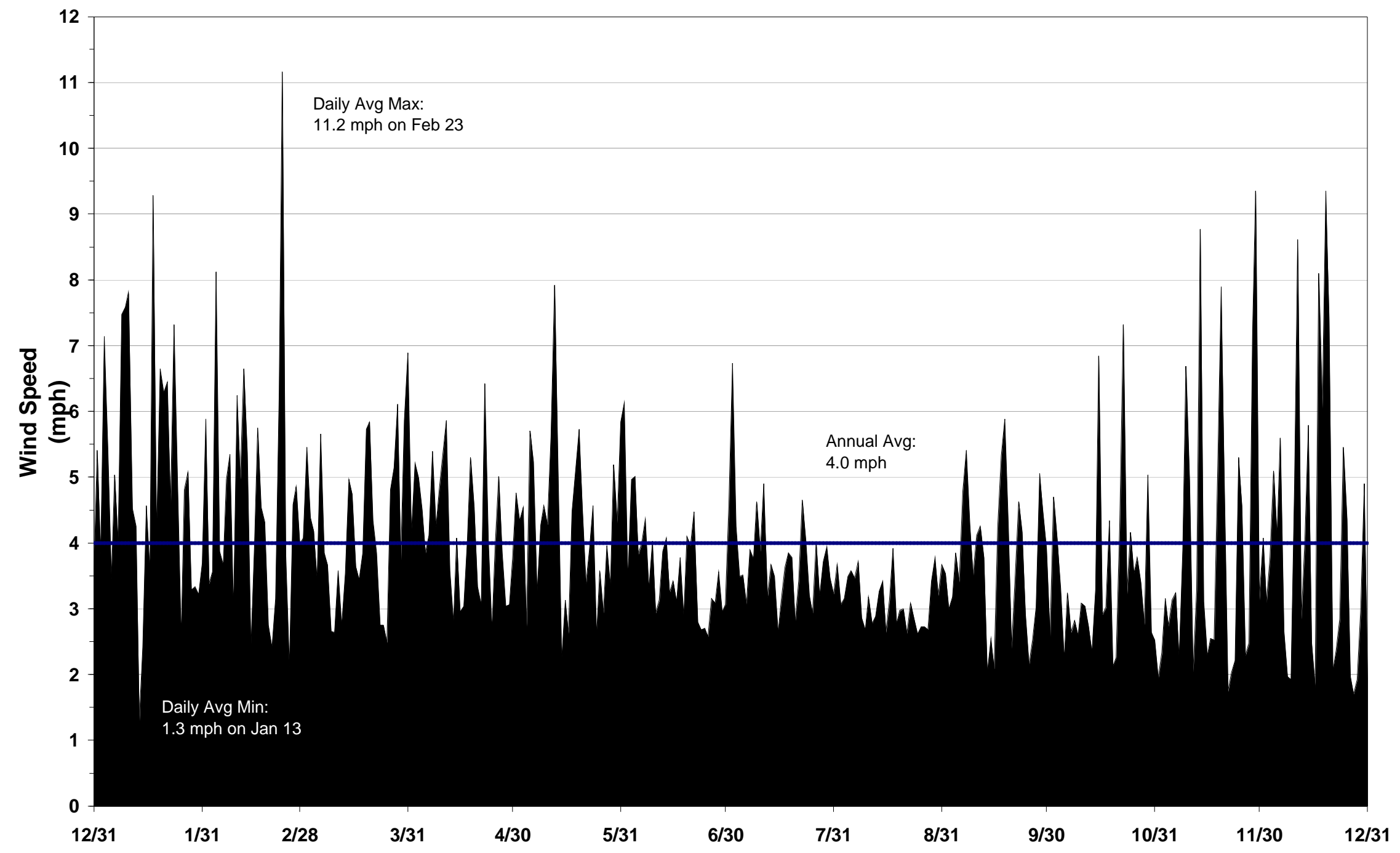


Fig. 14(a) - Annual Wind Rose Plots for 2003, 61-m Level

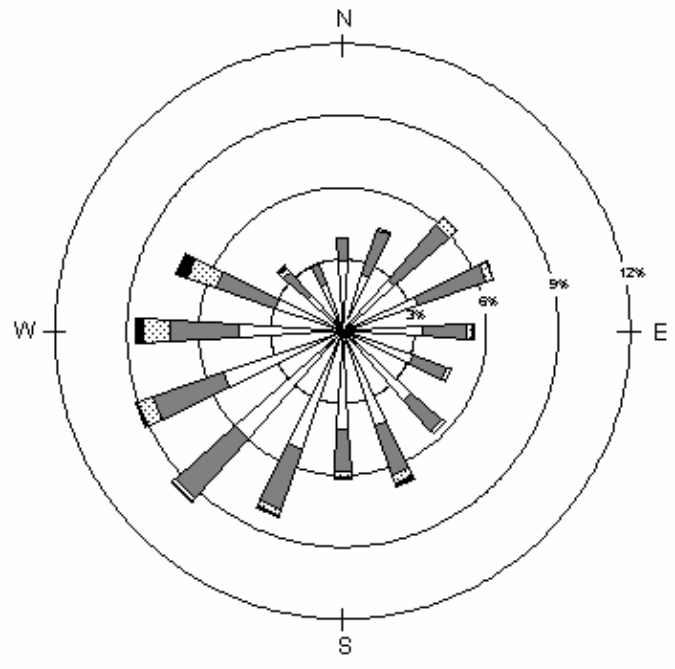

A Area Tower

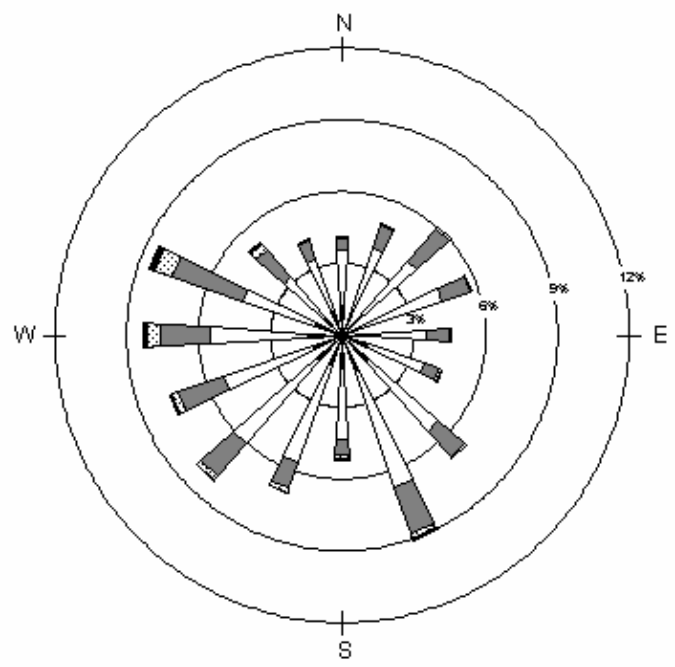

D Area Tower

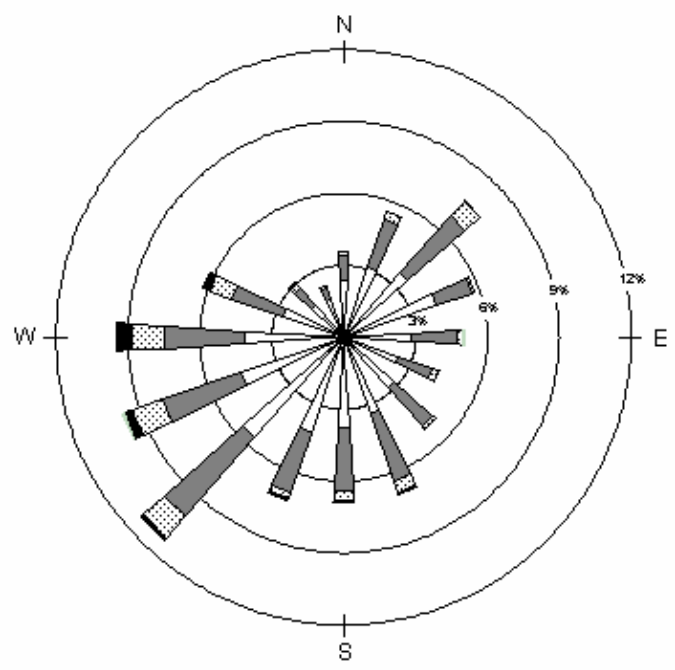

C Area Tower

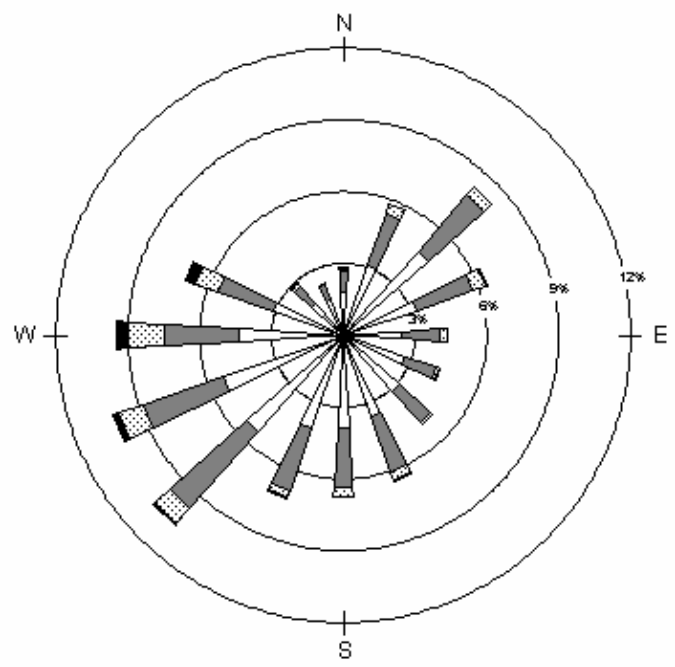

F Area Tower

\section{$\begin{array}{llllll}0.0 & 2.0 & 4.0 & 6.0 & 8.0 & 12.0\end{array}$}

Wind Speed Class Boundaries (Metersisecond)

Wind rose plots depict the frequency of occurrence of wind direction sector (direction from which the wind blows) by wind speed category. 
Fig. 14(b) - Annual Wind Rose Plots for 2003, 61-m Level

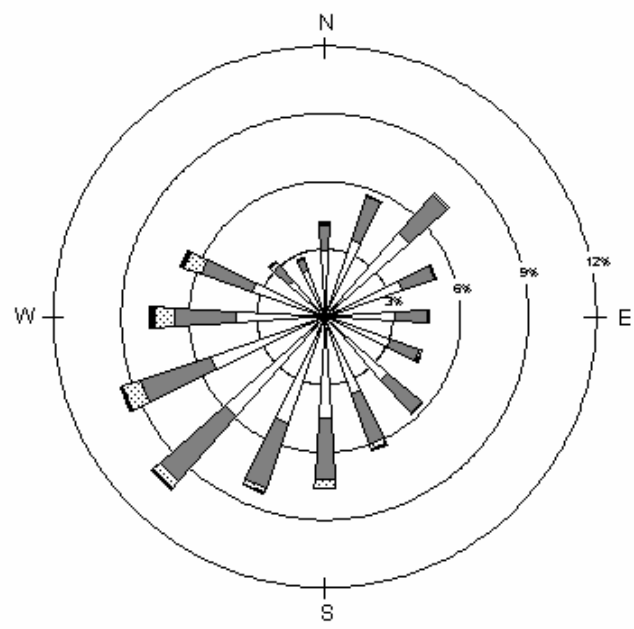

H Area Tower

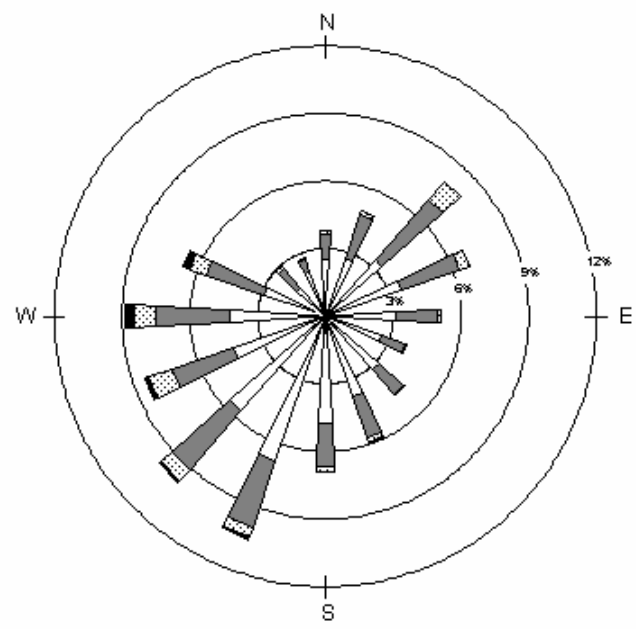

$\mathrm{L}$ Area Tower

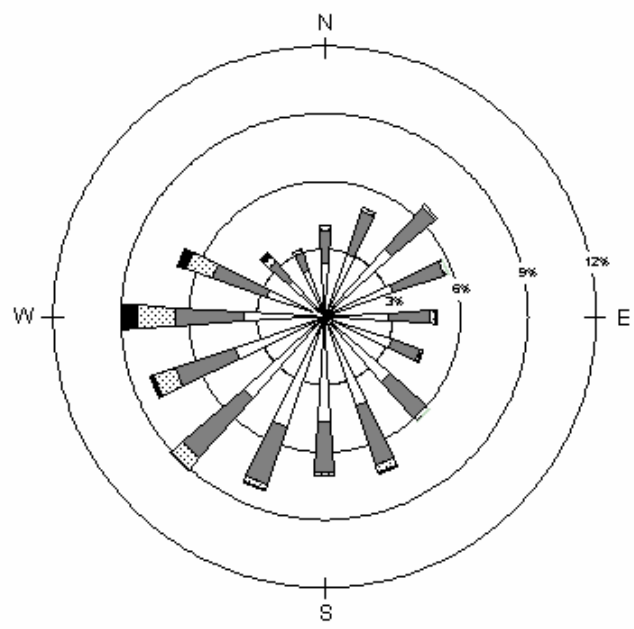

K Area Tower

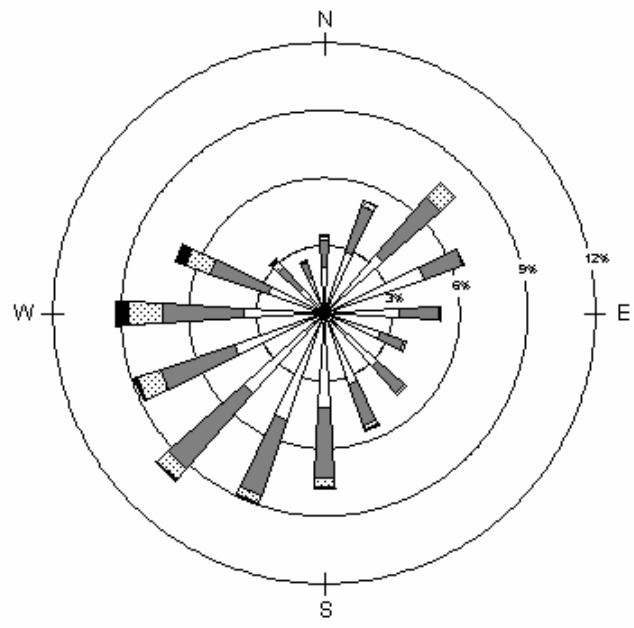

P Area Tower

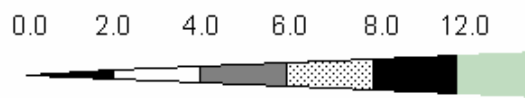

Wind Speed Class Boundaries (M etersisecond)

Wind rose plots depict the frequency of occurrence of wind direction sector (direction from which the wind blows) by wind speed category. 
Fig. 15 - Annual Wind Rose Plots for 2003, Central Climatology, All Levels

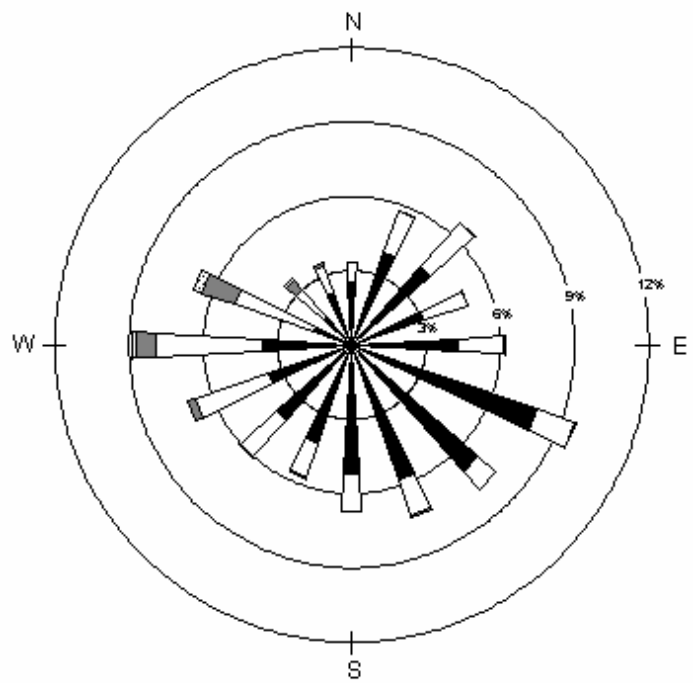

$4 \mathrm{~m}$ Level

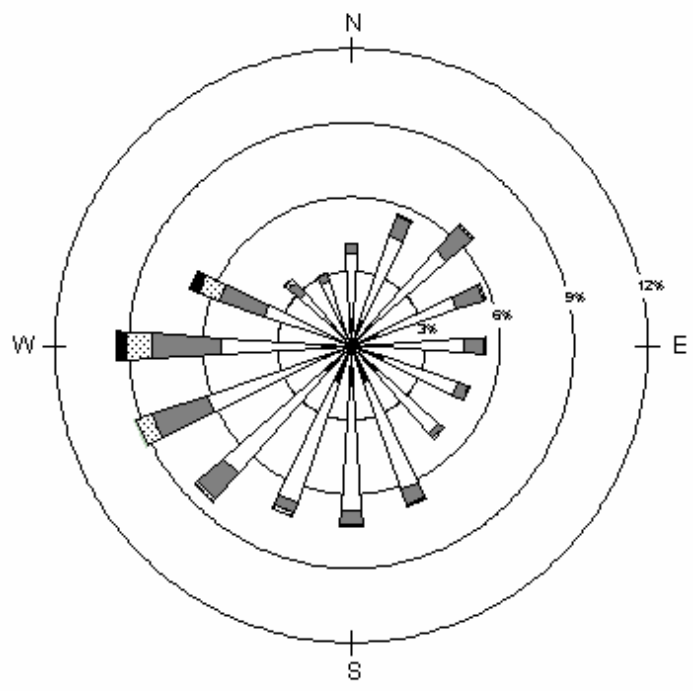

$36 \mathrm{~m}$ Level

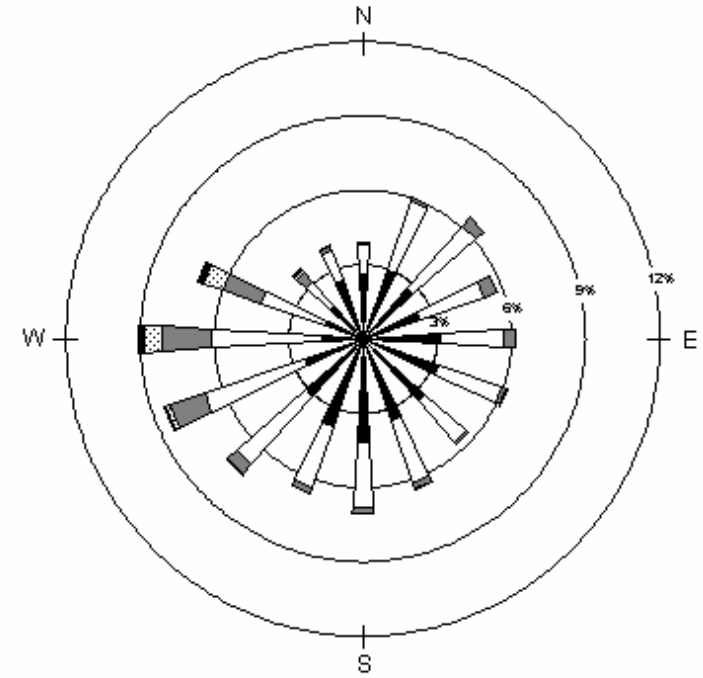

$18 \mathrm{~m}$ Level

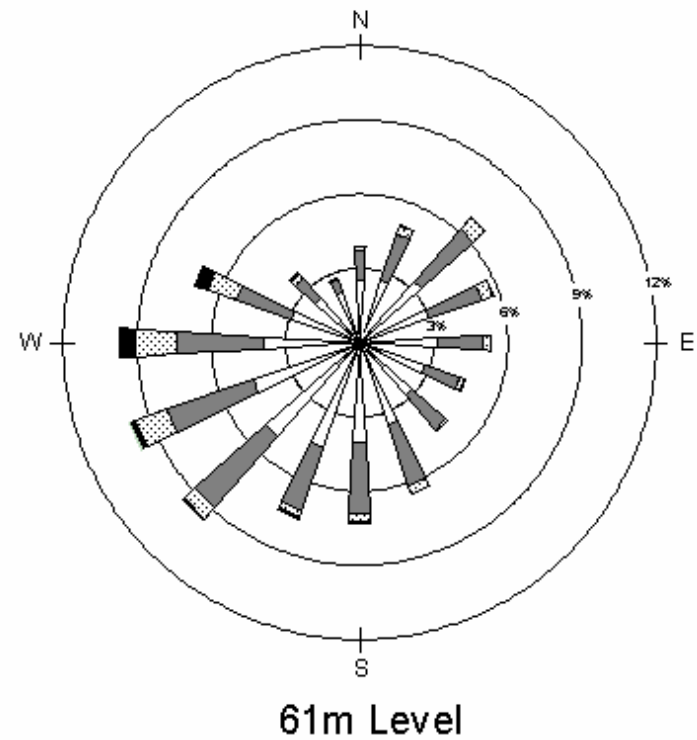

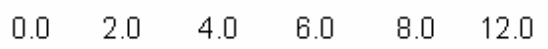

Wind Speed Class Boundaries (M etersisecond)

Wind rose plots depict the frequency of occurrence of wind direction sector (direction from which the wind blows) by wind speed category. 
Fig. 16 - Seasonal Wind Rose Plots for 2003, Central Climatology, 61-m Level

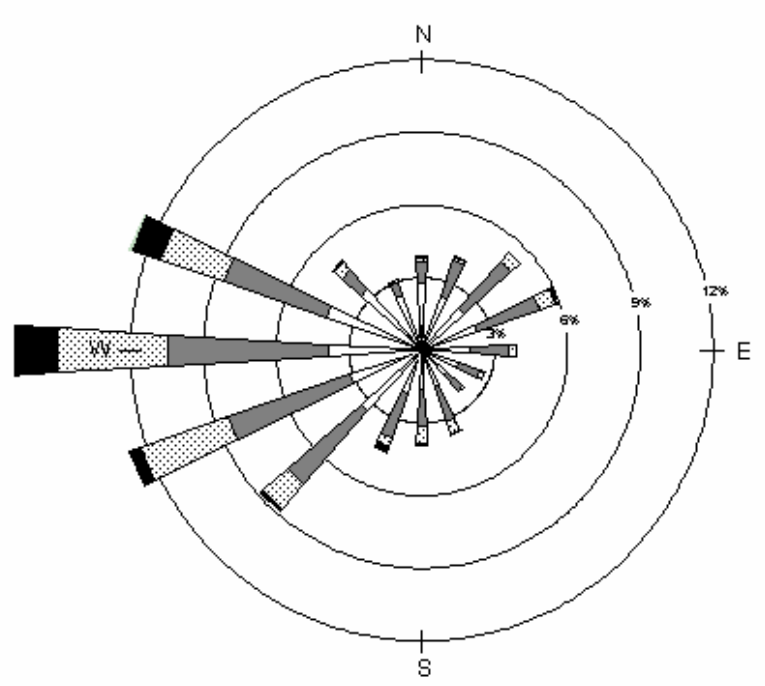

Winter

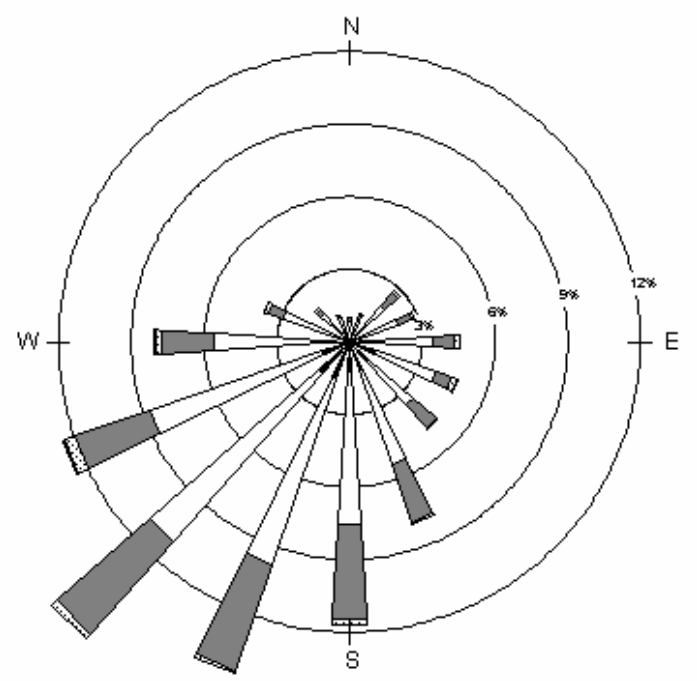

Summer

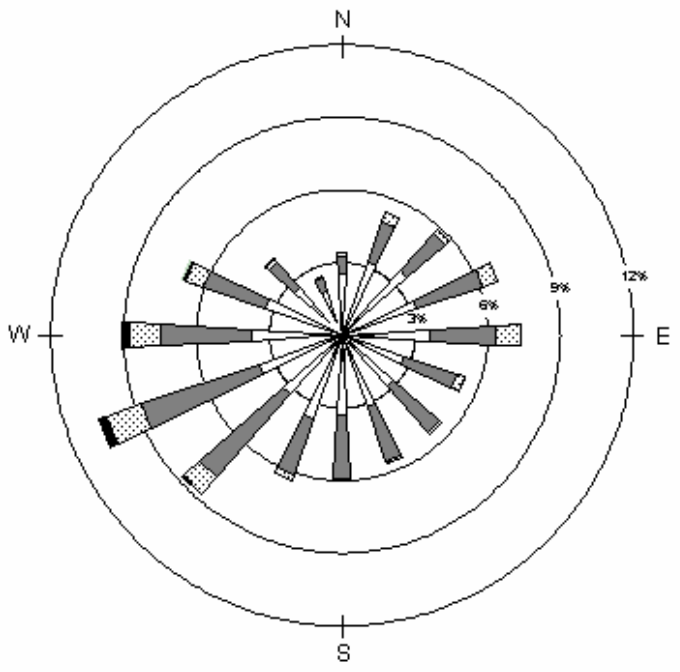

Spring

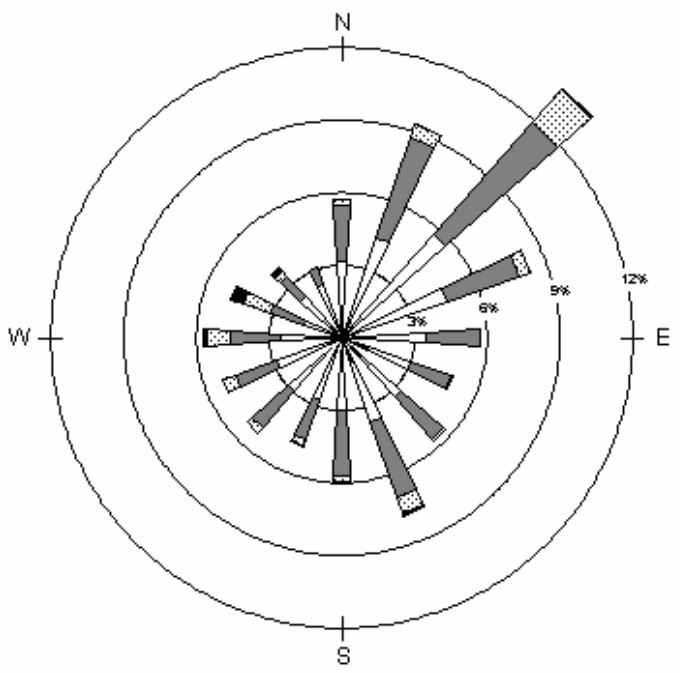

Fall

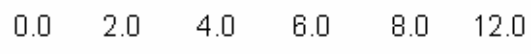

Wind Speed Class Boundaries

(Metersisecond)

Wind rose plots depict the frequency of occurrence of wind direction sector (direction from which the wind blows) by wind speed category. 
Fig. 17 - Daily Average Barometric Pressure for 2003

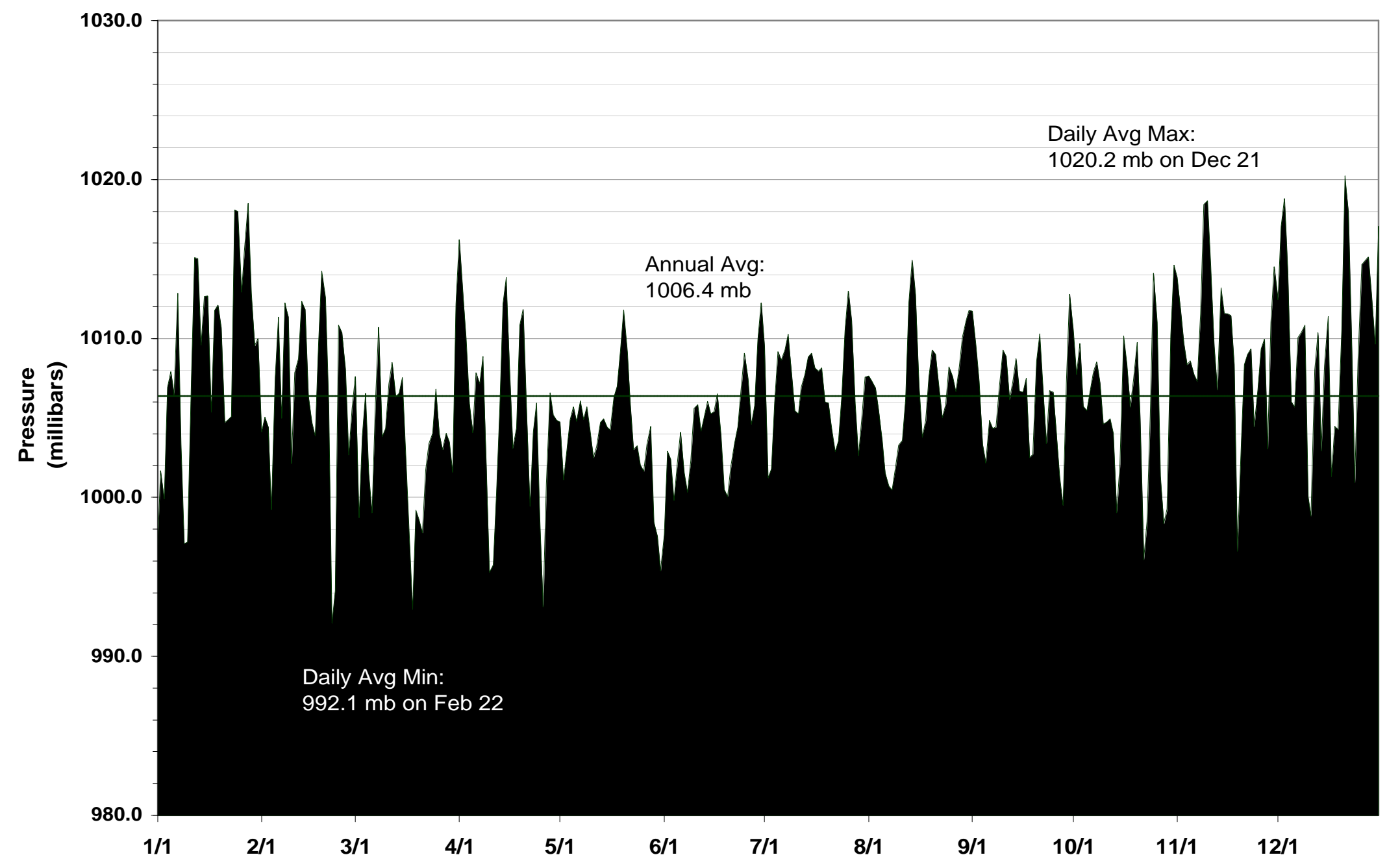


Fig. 18 - Daily Solar Radiation for 2003

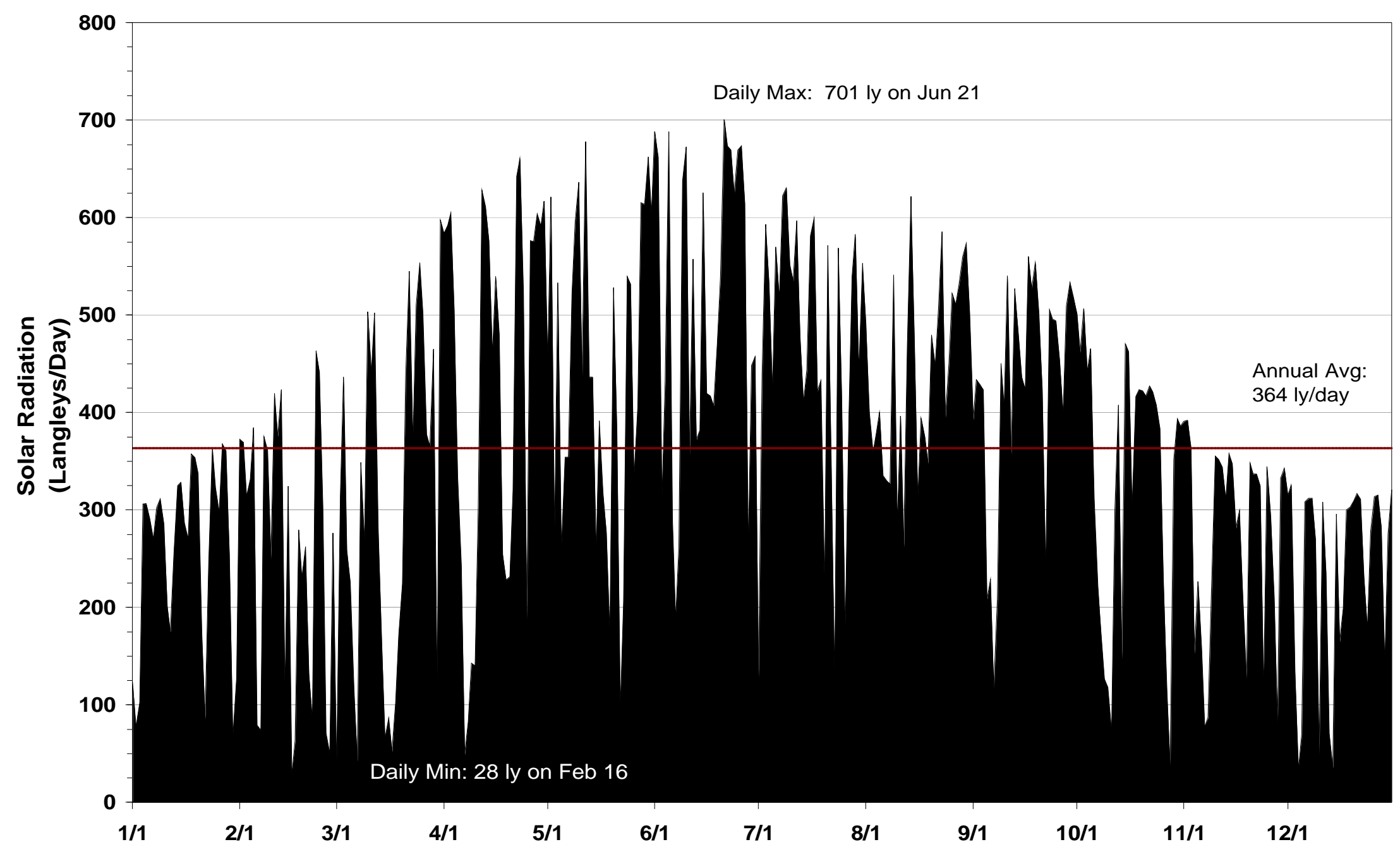


Table A.1 - Joint Occurrence Frequencies of Wind Direction Sector by Wind Speed Category for the A Area Tower, 2003

\begin{tabular}{|c|c|c|c|c|c|c|c|}
\hline \multirow[b]{2}{*}{ Sector } & \multicolumn{6}{|c|}{ Wind Speed Category, meters/sec } & \multirow[b]{2}{*}{ Total } \\
\hline & $0-2$ & $2-4$ & $4-6$ & $6-8$ & 8-12 & $>12$ & \\
\hline $\mathbf{N}$ & 0.00715 & 0.02265 & 0.00895 & 0.00037 & 0.00003 & 0.00000 & 0.03916 \\
\hline NNE & 0.00561 & 0.01942 & 0.01971 & 0.00094 & 0.00000 & 0.00000 & 0.04568 \\
\hline NE & 0.00501 & 0.02340 & 0.03075 & 0.00461 & 0.00006 & 0.00000 & 0.06381 \\
\hline ENE & 0.00489 & 0.02834 & 0.03015 & 0.00329 & 0.00020 & 0.00000 & 0.06687 \\
\hline E & 0.00549 & 0.02789 & 0.01833 & 0.00277 & 0.00020 & 0.00000 & 0.05468 \\
\hline ESE & 0.00621 & 0.02477 & 0.01542 & 0.00123 & 0.00014 & 0.00003 & 0.04779 \\
\hline SE & 0.00778 & 0.03052 & 0.01699 & 0.00169 & 0.00020 & 0.00003 & 0.05720 \\
\hline SSE & 0.00827 & 0.03346 & 0.02159 & 0.00352 & 0.00132 & 0.00003 & 0.06819 \\
\hline s & 0.00727 & 0.03392 & 0.01719 & 0.00249 & 0.00060 & 0.00000 & 0.06146 \\
\hline ssw & 0.00801 & 0.04410 & 0.02646 & 0.00283 & 0.00032 & 0.00003 & 0.08174 \\
\hline sw & 0.00972 & 0.04994 & 0.03289 & 0.00209 & 0.00020 & 0.00000 & 0.09484 \\
\hline WSW & 0.00944 & 0.04299 & 0.03138 & 0.00646 & 0.00106 & 0.00003 & 0.09135 \\
\hline WSW & 0.00755 & 0.03589 & 0.02897 & 0.01121 & 0.00312 & 0.00009 & 0.08683 \\
\hline WNW & 0.00646 & 0.02345 & 0.02566 & 0.01284 & 0.00495 & 0.00003 & 0.07339 \\
\hline NW & 0.00569 & 0.01424 & 0.01299 & 0.00246 & 0.00129 & 0.00000 & 0.03667 \\
\hline NNW & 0.00626 & 0.01487 & 0.00847 & 0.00063 & 0.00011 & 0.00000 & 0.03035 \\
\hline Total & 0.11080 & 0.46985 & 0.34587 & 0.05943 & 0.01379 & 0.00026 & 1.00000 \\
\hline
\end{tabular}

Table A.2 - Joint Occurrence Frequencies of Wind Direction Sector by Wind Speed Category for the C Area Tower, 2003

\begin{tabular}{|c|c|c|c|c|c|c|c|}
\hline \multirow[b]{2}{*}{ Sector } & \multicolumn{6}{|c|}{ Wind Speed Category, meters/sec } & \multirow[b]{2}{*}{ Total } \\
\hline & $0-2$ & $2-4$ & 4-6 & $6-8$ & $8-12$ & $>12$ & \\
\hline $\mathbf{N}$ & 0.00514 & 0.01879 & 0.01002 & 0.00154 & 0.00023 & 0.00000 & 0.03572 \\
\hline NNE & 0.00725 & 0.02350 & 0.02161 & 0.00297 & 0.00003 & 0.00000 & 0.05536 \\
\hline NE & 0.00654 & 0.02929 & 0.03161 & 0.00822 & 0.00034 & 0.00000 & 0.07600 \\
\hline ENE & 0.00691 & 0.03378 & 0.01616 & 0.00100 & 0.00000 & 0.00000 & 0.05784 \\
\hline E & 0.00540 & 0.02313 & 0.01867 & 0.00288 & 0.00009 & 0.00003 & 0.05019 \\
\hline ESE & 0.00568 & 0.01836 & 0.01508 & 0.00286 & 0.00017 & 0.00003 & 0.04217 \\
\hline SE & 0.00620 & 0.02207 & 0.02096 & 0.00208 & 0.00006 & 0.00000 & 0.05136 \\
\hline SSE & 0.00745 & 0.02661 & 0.02952 & 0.00474 & 0.00086 & 0.00003 & 0.06921 \\
\hline s & 0.00782 & 0.02958 & 0.02684 & 0.00394 & 0.00054 & 0.00000 & 0.06872 \\
\hline ssw & 0.00834 & 0.03372 & 0.02627 & 0.00300 & 0.00066 & 0.00000 & 0.07198 \\
\hline sw & 0.00908 & 0.04662 & 0.04411 & 0.01185 & 0.00126 & 0.00011 & 0.11303 \\
\hline WSW & 0.00765 & 0.03749 & 0.03472 & 0.01336 & 0.00403 & 0.00026 & 0.09750 \\
\hline WSW & 0.00765 & 0.03363 & 0.03400 & 0.01296 & 0.00660 & 0.00029 & 0.09513 \\
\hline WNW & 0.00622 & 0.02044 & 0.02284 & 0.00931 & 0.00351 & 0.00000 & 0.06233 \\
\hline NW & 0.00491 & 0.01245 & 0.01056 & 0.00194 & 0.00103 & 0.00000 & 0.03089 \\
\hline NNW & 0.00488 & 0.01173 & 0.00537 & 0.00054 & 0.00006 & 0.00000 & 0.02258 \\
\hline Total & 0.10712 & 0.42117 & 0.36833 & 0.08319 & 0.01944 & 0.00074 & 1.00000 \\
\hline
\end{tabular}


Table A.3 - Joint Occurrence Frequencies of Wind Direction Sector by Wind Speed Category for the D Area Tower, 2003

\begin{tabular}{|c|c|c|c|c|c|c|c|}
\hline \multirow[b]{2}{*}{ Sector } & \multicolumn{6}{|c|}{ Wind Speed Category, meters/sec } & \multirow[b]{2}{*}{ Total } \\
\hline & $0-2$ & 2-4 & 4-6 & 6-8 & 8-12 & $>12$ & \\
\hline $\mathbf{N}$ & 0.01273 & 0.02329 & 0.00457 & 0.00040 & 0.00003 & 0.00000 & 0.04101 \\
\hline NNE & 0.00905 & 0.02948 & 0.01033 & 0.00049 & 0.00000 & 0.00000 & 0.04934 \\
\hline NE & 0.00796 & 0.03328 & 0.01829 & 0.00117 & 0.00000 & 0.00000 & 0.06070 \\
\hline ENE & 0.00876 & 0.03527 & 0.01244 & 0.00069 & 0.00000 & 0.00000 & 0.05716 \\
\hline E & 0.01025 & 0.02474 & 0.00956 & 0.00074 & 0.00000 & 0.00003 & 0.04532 \\
\hline ESE & 0.01187 & 0.02429 & 0.00694 & 0.00089 & 0.00011 & 0.00000 & 0.04409 \\
\hline SE & 0.01515 & 0.03867 & 0.01433 & 0.00077 & 0.00014 & 0.00006 & 0.06912 \\
\hline SSE & 0.01607 & 0.05117 & 0.01995 & 0.00231 & 0.00103 & 0.00003 & 0.09055 \\
\hline s & 0.01393 & 0.02948 & 0.00622 & 0.00191 & 0.00103 & 0.00000 & 0.05257 \\
\hline ssw & 0.01333 & 0.04292 & 0.01124 & 0.00206 & 0.00037 & 0.00000 & 0.06992 \\
\hline sw & 0.01416 & 0.04652 & 0.01812 & 0.00188 & 0.00029 & 0.00000 & 0.08096 \\
\hline wsw & 0.01267 & 0.03941 & 0.02018 & 0.00305 & 0.00094 & 0.00000 & 0.07626 \\
\hline wsw & 0.01416 & 0.04070 & 0.02120 & 0.00591 & 0.00103 & 0.00000 & 0.08299 \\
\hline WNW & 0.01187 & 0.03194 & 0.03102 & 0.00782 & 0.00243 & 0.00000 & 0.08508 \\
\hline NW & 0.01065 & 0.02175 & 0.01507 & 0.00345 & 0.00134 & 0.00003 & 0.05228 \\
\hline NNW & 0.01079 & 0.02255 & 0.00825 & 0.00089 & 0.00017 & 0.00000 & 0.04264 \\
\hline Total & 0.19338 & 0.53545 & 0.22771 & 0.03442 & 0.00890 & 0.00014 & 1.00000 \\
\hline
\end{tabular}

Table A.4 - Joint Occurrence Frequencies of Wind Direction Sector by Wind Speed Category for the F Area Tower, 2003

\begin{tabular}{|c|c|c|c|c|c|c|c|}
\hline \multirow[b]{2}{*}{ Sector } & \multicolumn{6}{|c|}{ Wind Speed Category, meters/sec } & \multirow[b]{2}{*}{ Total } \\
\hline & $0-2$ & $2-4$ & 4-6 & 6-8 & 8-12 & $>12$ & \\
\hline $\mathbf{N}$ & 0.00474 & 0.01373 & 0.00813 & 0.00140 & 0.00026 & 0.00000 & 0.02826 \\
\hline NNE & 0.00679 & 0.02435 & 0.02272 & 0.00437 & 0.00011 & 0.00000 & 0.05834 \\
\hline NE & 0.00714 & 0.03987 & 0.03125 & 0.00420 & 0.00020 & 0.00000 & 0.08265 \\
\hline ENE & 0.00651 & 0.02554 & 0.02500 & 0.00579 & 0.00071 & 0.00000 & 0.06356 \\
\hline E & 0.00554 & 0.01921 & 0.01595 & 0.00308 & 0.00011 & 0.00003 & 0.04392 \\
\hline ESE & 0.00642 & 0.02095 & 0.01364 & 0.00151 & 0.00029 & 0.00006 & 0.04287 \\
\hline SE & 0.00656 & 0.02446 & 0.01775 & 0.00109 & 0.00003 & 0.00000 & 0.04989 \\
\hline SSE & 0.00711 & 0.02903 & 0.02409 & 0.00357 & 0.00086 & 0.00006 & 0.06470 \\
\hline s & 0.00659 & 0.03188 & 0.02526 & 0.00363 & 0.00057 & 0.00000 & 0.06793 \\
\hline ssw & 0.00862 & 0.03251 & 0.02740 & 0.00300 & 0.00100 & 0.00000 & 0.07252 \\
\hline sw & 0.00905 & 0.04504 & 0.04278 & 0.00816 & 0.00097 & 0.00003 & 0.10603 \\
\hline wsw & 0.01005 & 0.04307 & 0.03511 & 0.01133 & 0.00191 & 0.00006 & 0.10152 \\
\hline wsw & 0.00891 & 0.03479 & 0.03148 & 0.01487 & 0.00480 & 0.00026 & 0.09510 \\
\hline WNW & 0.00731 & 0.02338 & 0.02446 & 0.01022 & 0.00428 & 0.00000 & 0.06964 \\
\hline NW & 0.00571 & 0.01196 & 0.00959 & 0.00174 & 0.00106 & 0.00000 & 0.03005 \\
\hline NNW & 0.00468 & 0.01127 & 0.00651 & 0.00049 & 0.00009 & 0.00000 & 0.02303 \\
\hline Total & 0.11171 & 0.43102 & 0.36112 & 0.07843 & 0.01724 & 0.00049 & 1.00001 \\
\hline
\end{tabular}


Table A.5 - Joint Occurrence Frequencies of Wind Direction Sector by Wind Speed Category for the H Area Tower, 2003

\begin{tabular}{|c|c|c|c|c|c|c|c|}
\hline \multirow[b]{2}{*}{ Sector } & \multicolumn{6}{|c|}{ Wind Speed Category, meters/sec } & \multirow[b]{2}{*}{ Total } \\
\hline & $0-2$ & 2-4 & 4-6 & 6-8 & 8-12 & $>12$ & \\
\hline $\mathbf{N}$ & 0.00714 & 0.02192 & 0.01159 & 0.00094 & 0.00023 & 0.00000 & 0.04181 \\
\hline NNE & 0.00674 & 0.02917 & 0.02032 & 0.00077 & 0.00000 & 0.00000 & 0.05700 \\
\hline NE & 0.00833 & 0.04116 & 0.02255 & 0.00111 & 0.00003 & 0.00000 & 0.07318 \\
\hline ENE & 0.00731 & 0.02917 & 0.01507 & 0.00060 & 0.00000 & 0.00000 & 0.05214 \\
\hline E & 0.00648 & 0.02472 & 0.01379 & 0.00083 & 0.00000 & 0.00003 & 0.04584 \\
\hline ESE & 0.00662 & 0.02389 & 0.01390 & 0.00103 & 0.00020 & 0.00000 & 0.04564 \\
\hline SE & 0.00876 & 0.02860 & 0.01966 & 0.00051 & 0.00009 & 0.00000 & 0.05762 \\
\hline SSE & 0.00742 & 0.02868 & 0.02377 & 0.00228 & 0.00074 & 0.00000 & 0.06290 \\
\hline s & 0.00782 & 0.03673 & 0.02788 & 0.00334 & 0.00046 & 0.00000 & 0.07623 \\
\hline ssw & 0.00853 & 0.04176 & 0.02980 & 0.00228 & 0.00069 & 0.00000 & 0.08305 \\
\hline sw & 0.00828 & 0.05086 & 0.03819 & 0.00445 & 0.00069 & 0.00000 & 0.10246 \\
\hline wsw & 0.00813 & 0.04509 & 0.03276 & 0.00856 & 0.00111 & 0.00006 & 0.09572 \\
\hline wsw & 0.00645 & 0.03311 & 0.02729 & 0.00848 & 0.00263 & 0.00000 & 0.07795 \\
\hline WNW & 0.00716 & 0.02714 & 0.02340 & 0.00805 & 0.00197 & 0.00000 & 0.06773 \\
\hline NW & 0.00591 & 0.01530 & 0.00899 & 0.00206 & 0.00063 & 0.00000 & 0.03288 \\
\hline NNW & 0.00608 & 0.01595 & 0.00514 & 0.00063 & 0.00006 & 0.00000 & 0.02786 \\
\hline Total & 0.11716 & 0.49324 & 0.33409 & 0.04592 & 0.00951 & 0.00009 & 1.00000 \\
\hline
\end{tabular}

Table A.6 - Joint Occurrence Frequencies of Wind Direction Sector by Wind Speed Category for the K Area Tower, 2003

\begin{tabular}{|c|c|c|c|c|c|c|c|}
\hline \multirow[b]{2}{*}{ Sector } & \multicolumn{6}{|c|}{ Wind Speed Category, meters/sec } & \multirow[b]{2}{*}{ Total } \\
\hline & $0-2$ & $2-4$ & 4-6 & 6-8 & 8-12 & $>12$ & \\
\hline $\mathbf{N}$ & 0.00505 & 0.01891 & 0.01446 & 0.00187 & 0.00017 & 0.00000 & 0.04046 \\
\hline NNE & 0.00505 & 0.02433 & 0.02029 & 0.00158 & 0.00000 & 0.00000 & 0.05125 \\
\hline NE & 0.00669 & 0.03354 & 0.02459 & 0.00158 & 0.00003 & 0.00000 & 0.06643 \\
\hline ENE & 0.00594 & 0.02660 & 0.02327 & 0.00212 & 0.00009 & 0.00003 & 0.05805 \\
\hline E & 0.00539 & 0.02313 & 0.01842 & 0.00244 & 0.00006 & 0.00003 & 0.04947 \\
\hline ESE & 0.00729 & 0.02376 & 0.01352 & 0.00132 & 0.00020 & 0.00003 & 0.04611 \\
\hline SE & 0.00786 & 0.03030 & 0.02175 & 0.00161 & 0.00003 & 0.00003 & 0.06158 \\
\hline SSE & 0.00760 & 0.03406 & 0.02801 & 0.00307 & 0.00078 & 0.00003 & 0.07354 \\
\hline s & 0.00792 & 0.03831 & 0.02258 & 0.00189 & 0.00014 & 0.00000 & 0.07084 \\
\hline ssw & 0.00947 & 0.04247 & 0.02683 & 0.00250 & 0.00023 & 0.00000 & 0.08149 \\
\hline sw & 0.00809 & 0.04006 & 0.03564 & 0.00654 & 0.00089 & 0.00000 & 0.09122 \\
\hline wsw & 0.00720 & 0.03458 & 0.02778 & 0.01042 & 0.00155 & 0.00000 & 0.08152 \\
\hline wsw & 0.00697 & 0.02884 & 0.03044 & 0.01659 & 0.00628 & 0.00009 & 0.08921 \\
\hline WNW & 0.00557 & 0.02135 & 0.02562 & 0.01188 & 0.00465 & 0.00006 & 0.06912 \\
\hline NW & 0.00620 & 0.01512 & 0.01145 & 0.00353 & 0.00138 & 0.00006 & 0.03773 \\
\hline NNW & 0.00542 & 0.01613 & 0.00824 & 0.00181 & 0.00040 & 0.00000 & 0.03199 \\
\hline Total & 0.10772 & 0.45147 & 0.35287 & 0.07073 & 0.01687 & 0.00035 & 1.00000 \\
\hline
\end{tabular}


Table A.7 - Joint Occurrence Frequencies of Wind Direction Sector by Wind Speed Category for the L Area Tower, 2003

\begin{tabular}{|c|c|c|c|c|c|c|c|}
\hline \multirow[b]{2}{*}{ Sector } & \multicolumn{6}{|c|}{ Wind Speed Category, meters/sec } & \multirow[b]{2}{*}{ Total } \\
\hline & $0-2$ & $2-4$ & $4-6$ & $6-8$ & 8-12 & $>12$ & \\
\hline $\mathbf{N}$ & 0.00654 & 0.01910 & 0.01116 & 0.00106 & 0.00003 & 0.00000 & 0.03788 \\
\hline NNE & 0.00468 & 0.02215 & 0.02089 & 0.00194 & 0.00006 & 0.00000 & 0.04972 \\
\hline NE & 0.00522 & 0.02820 & 0.03548 & 0.01093 & 0.00020 & 0.00000 & 0.08004 \\
\hline ENE & 0.00568 & 0.03020 & 0.02680 & 0.00468 & 0.00014 & 0.00000 & 0.06751 \\
\hline E & 0.00617 & 0.02489 & 0.01824 & 0.00154 & 0.00003 & 0.00006 & 0.05092 \\
\hline ESE & 0.00668 & 0.01972 & 0.01085 & 0.00046 & 0.00009 & 0.00000 & 0.03779 \\
\hline SE & 0.00748 & 0.02415 & 0.01436 & 0.00071 & 0.00003 & 0.00000 & 0.04673 \\
\hline SSE & 0.00779 & 0.02963 & 0.01958 & 0.00211 & 0.00071 & 0.00000 & 0.05983 \\
\hline s & 0.00762 & 0.04013 & 0.01864 & 0.00251 & 0.00054 & 0.00000 & 0.06945 \\
\hline ssw & 0.00976 & 0.05832 & 0.03091 & 0.00405 & 0.00168 & 0.00000 & 0.10473 \\
\hline sw & 0.00828 & 0.04807 & 0.03442 & 0.00651 & 0.00103 & 0.00006 & 0.09836 \\
\hline WSW & 0.00702 & 0.03585 & 0.02857 & 0.01096 & 0.00234 & 0.00003 & 0.08478 \\
\hline WSW & 0.00668 & 0.03579 & 0.03288 & 0.00942 & 0.00368 & 0.00009 & 0.08854 \\
\hline WNW & 0.00520 & 0.02346 & 0.02706 & 0.00796 & 0.00291 & 0.00000 & 0.06659 \\
\hline NW & 0.00417 & 0.01250 & 0.01119 & 0.00151 & 0.00060 & 0.00000 & 0.02997 \\
\hline NNW & 0.00545 & 0.01547 & 0.00579 & 0.00037 & 0.00009 & 0.00000 & 0.02717 \\
\hline Total & 0.10441 & 0.46763 & 0.34683 & 0.06674 & 0.01416 & 0.00023 & 1.00000 \\
\hline
\end{tabular}

Table A.8 - Joint Occurrence Frequencies of Wind Direction Sector by Wind Speed Category for the P Area Tower, 2003

\begin{tabular}{|c|c|c|c|c|c|c|c|}
\hline \multirow[b]{2}{*}{ Sector } & \multicolumn{6}{|c|}{ Wind Speed Category, meters/sec } & \multirow[b]{2}{*}{ Total } \\
\hline & $0-2$ & $2-4$ & 4-6 & $6-8$ & 8-12 & $>12$ & \\
\hline $\mathbf{N}$ & 0.00374 & 0.01641 & 0.01216 & 0.00217 & 0.00009 & 0.00000 & 0.03456 \\
\hline NNE & 0.00605 & 0.02249 & 0.02143 & 0.00271 & 0.00006 & 0.00000 & 0.05274 \\
\hline NE & 0.00639 & 0.02803 & 0.03405 & 0.00882 & 0.00034 & 0.00000 & 0.07763 \\
\hline ENE & 0.00893 & 0.03784 & 0.01807 & 0.00080 & 0.00000 & 0.00000 & 0.06564 \\
\hline E & 0.00742 & 0.02557 & 0.01738 & 0.00128 & 0.00011 & 0.00000 & 0.05177 \\
\hline ESE & 0.00648 & 0.01995 & 0.01082 & 0.00114 & 0.00014 & 0.00003 & 0.03856 \\
\hline SE & 0.00802 & 0.02369 & 0.01578 & 0.00066 & 0.00009 & 0.00000 & 0.04823 \\
\hline SSE & 0.00819 & 0.02543 & 0.01906 & 0.00203 & 0.00046 & 0.00000 & 0.05517 \\
\hline s & 0.00856 & 0.03365 & 0.03091 & 0.00400 & 0.00086 & 0.00000 & 0.07797 \\
\hline ssw & 0.00836 & 0.04152 & 0.03573 & 0.00328 & 0.00089 & 0.00000 & 0.08978 \\
\hline sw & 0.00679 & 0.04075 & 0.04486 & 0.00645 & 0.00097 & 0.00000 & 0.09983 \\
\hline WSW & 0.00662 & 0.03542 & 0.03453 & 0.01073 & 0.00154 & 0.00000 & 0.08884 \\
\hline WSW & 0.00614 & 0.02920 & 0.03644 & 0.01513 & 0.00522 & 0.00017 & 0.09230 \\
\hline WNW & 0.00494 & 0.02109 & 0.02731 & 0.01164 & 0.00491 & 0.00000 & 0.06989 \\
\hline NW & 0.00351 & 0.01050 & 0.01293 & 0.00360 & 0.00186 & 0.00009 & 0.03248 \\
\hline NNW & 0.00351 & 0.01281 & 0.00742 & 0.00080 & 0.00009 & 0.00000 & 0.02463 \\
\hline Total & 0.10365 & 0.42434 & 0.37888 & 0.07523 & 0.01761 & 0.00029 & 1.00000 \\
\hline
\end{tabular}


Table A.9 - Joint Occurrence Frequencies of Wind Direction Sector by Wind Speed Category for the 2m Level Central Climatology Tower, 2003

\begin{tabular}{|c|c|c|c|c|c|c|c|}
\hline \multirow[b]{2}{*}{ Sector } & \multicolumn{6}{|c|}{ Wind Speed Category, meters/sec } & \multirow[b]{2}{*}{ Total } \\
\hline & $0-2$ & $2-4$ & $4-6$ & $6-8$ & $8-12$ & $>12$ & \\
\hline $\mathbf{N}$ & 0.02581 & 0.00722 & 0.00037 & 0.00000 & 0.00000 & 0.00000 & 0.03341 \\
\hline NNE & 0.03935 & 0.01770 & 0.00011 & 0.00003 & 0.00000 & 0.00000 & 0.05719 \\
\hline NE & 0.04243 & 0.02359 & 0.00034 & 0.00000 & 0.00000 & 0.00000 & 0.06636 \\
\hline ENE & 0.03072 & 0.01847 & 0.00049 & 0.00000 & 0.00000 & 0.00000 & 0.04968 \\
\hline E & 0.04263 & 0.01859 & 0.00094 & 0.00003 & 0.00000 & 0.00000 & 0.06219 \\
\hline ESE & 0.07926 & 0.01605 & 0.00049 & 0.00009 & 0.00000 & 0.00000 & 0.09588 \\
\hline SE & 0.06790 & 0.00985 & 0.00011 & 0.00000 & 0.00000 & 0.00000 & 0.07787 \\
\hline SSE & 0.05785 & 0.01502 & 0.00083 & 0.00009 & 0.00000 & 0.00000 & 0.07378 \\
\hline s & 0.05237 & 0.01459 & 0.00060 & 0.00000 & 0.00000 & 0.00000 & 0.06756 \\
\hline ssw & 0.04226 & 0.01408 & 0.00080 & 0.00000 & 0.00000 & 0.00000 & 0.05714 \\
\hline sw & 0.04055 & 0.01956 & 0.00046 & 0.00000 & 0.00000 & 0.00000 & 0.06056 \\
\hline WSW & 0.03569 & 0.03124 & 0.00326 & 0.00009 & 0.00003 & 0.00000 & 0.07030 \\
\hline WSW & 0.03615 & 0.04277 & 0.00865 & 0.00106 & 0.00000 & 0.00000 & 0.08863 \\
\hline WNW & 0.01608 & 0.03384 & 0.01448 & 0.00348 & 0.00000 & 0.00000 & 0.06787 \\
\hline NW & 0.01511 & 0.01482 & 0.00503 & 0.00120 & 0.00003 & 0.00000 & 0.03618 \\
\hline NNW & 0.02224 & 0.01165 & 0.00149 & 0.00003 & 0.00000 & 0.00000 & 0.03541 \\
\hline Total & 0.64639 & 0.30903 & 0.03843 & 0.00608 & 0.00006 & 0.00000 & 1.00000 \\
\hline
\end{tabular}

Table A.10 - Joint Occurrence Frequencies of Wind Direction Sector by Wind Speed Category for the 18m Level Central Climatology Tower, 2003

\begin{tabular}{|c|c|c|c|c|c|c|c|}
\hline \multirow[b]{2}{*}{ Sector } & \multicolumn{6}{|c|}{ Wind Speed Category, meters/sec } & \multirow[b]{2}{*}{ Total } \\
\hline & $0-2$ & $2-4$ & 4-6 & $6-8$ & 8-12 & $>12$ & \\
\hline $\mathbf{N}$ & 0.01048 & 0.02684 & 0.00377 & 0.00031 & 0.00003 & 0.00000 & 0.04143 \\
\hline NNE & 0.01048 & 0.03669 & 0.00831 & 0.00046 & 0.00000 & 0.00000 & 0.05594 \\
\hline NE & 0.01216 & 0.03932 & 0.01362 & 0.00100 & 0.00000 & 0.00000 & 0.06610 \\
\hline ENE & 0.01194 & 0.03312 & 0.01151 & 0.00109 & 0.00000 & 0.00000 & 0.05765 \\
\hline E & 0.01148 & 0.03426 & 0.00765 & 0.00106 & 0.00003 & 0.00000 & 0.05448 \\
\hline ESE & 0.01422 & 0.03129 & 0.00474 & 0.00043 & 0.00006 & 0.00000 & 0.05074 \\
\hline SE & 0.01445 & 0.03284 & 0.00266 & 0.00011 & 0.00000 & 0.00000 & 0.05005 \\
\hline SSE & 0.01562 & 0.04583 & 0.00611 & 0.00077 & 0.00026 & 0.00000 & 0.06858 \\
\hline s & 0.01633 & 0.05034 & 0.00574 & 0.00083 & 0.00000 & 0.00000 & 0.07324 \\
\hline ssw & 0.01568 & 0.05105 & 0.00500 & 0.00129 & 0.00003 & 0.00000 & 0.07304 \\
\hline sw & 0.01411 & 0.05476 & 0.01416 & 0.00123 & 0.00017 & 0.00000 & 0.08443 \\
\hline WSW & 0.01228 & 0.04897 & 0.02416 & 0.00628 & 0.00106 & 0.00003 & 0.09277 \\
\hline WSW & 0.01196 & 0.04120 & 0.02775 & 0.01042 & 0.00383 & 0.00000 & 0.09517 \\
\hline WNW & 0.00865 & 0.02832 & 0.01944 & 0.00894 & 0.00400 & 0.00000 & 0.06935 \\
\hline NW & 0.01222 & 0.01588 & 0.00600 & 0.00166 & 0.00057 & 0.00000 & 0.03632 \\
\hline NNW & 0.01134 & 0.01613 & 0.00308 & 0.00014 & 0.00003 & 0.00000 & 0.03072 \\
\hline Total & 0.20338 & 0.58684 & 0.16369 & 0.03601 & 0.01005 & 0.00003 & 1.00000 \\
\hline
\end{tabular}


Table A.11 - Joint Occurrence Frequencies of Wind Direction Sector by Wind Speed Category for the 36m Level Central Climatology Tower, 2003

\begin{tabular}{|c|c|c|c|c|c|c|c|}
\hline \multirow[b]{2}{*}{ Sector } & \multicolumn{6}{|c|}{ Wind Speed Category, meters/sec } & \multirow[b]{2}{*}{ Total } \\
\hline & $0-2$ & $2-4$ & $4-6$ & $6-8$ & 8-12 & $>12$ & \\
\hline $\mathbf{N}$ & 0.01048 & 0.02684 & 0.00377 & 0.00031 & 0.00003 & 0.00000 & 0.04143 \\
\hline NNE & 0.01048 & 0.03669 & 0.00831 & 0.00046 & 0.00000 & 0.00000 & 0.05594 \\
\hline NE & 0.01216 & 0.03932 & 0.01362 & 0.00100 & 0.00000 & 0.00000 & 0.06610 \\
\hline ENE & 0.01194 & 0.03312 & 0.01151 & 0.00109 & 0.00000 & 0.00000 & 0.05765 \\
\hline E & 0.01148 & 0.03426 & 0.00765 & 0.00106 & 0.00003 & 0.00000 & 0.05448 \\
\hline ESE & 0.01422 & 0.03129 & 0.00474 & 0.00043 & 0.00006 & 0.00000 & 0.05074 \\
\hline SE & 0.01445 & 0.03284 & 0.00266 & 0.00011 & 0.00000 & 0.00000 & 0.05005 \\
\hline SSE & 0.01562 & 0.04583 & 0.00611 & 0.00077 & 0.00026 & 0.00000 & 0.06858 \\
\hline $\mathbf{S}$ & 0.01633 & 0.05034 & 0.00574 & 0.00083 & 0.00000 & 0.00000 & 0.07324 \\
\hline ssw & 0.01568 & 0.05105 & 0.00500 & 0.00129 & 0.00003 & 0.00000 & 0.07304 \\
\hline sw & 0.01411 & 0.05476 & 0.01416 & 0.00123 & 0.00017 & 0.00000 & 0.08443 \\
\hline WSW & 0.01228 & 0.04897 & 0.02416 & 0.00628 & 0.00106 & 0.00003 & 0.09277 \\
\hline WSW & 0.01196 & 0.04120 & 0.02775 & 0.01042 & 0.00383 & 0.00000 & 0.09517 \\
\hline WNW & 0.00865 & 0.02832 & 0.01944 & 0.00894 & 0.00400 & 0.00000 & 0.06935 \\
\hline NW & 0.01222 & 0.01588 & 0.00600 & 0.00166 & 0.00057 & 0.00000 & 0.03632 \\
\hline NNW & 0.01134 & 0.01613 & 0.00308 & 0.00014 & 0.00003 & 0.00000 & 0.03072 \\
\hline Total & 0.20338 & 0.58684 & 0.16369 & 0.03601 & 0.01005 & 0.00003 & 1.00000 \\
\hline
\end{tabular}

Table A.12 - Joint Occurrence Frequencies of Wind Direction Sector by Wind Speed Category for the 61m Level Central Climatology Tower, 2003

\begin{tabular}{|c|c|c|c|c|c|c|c|}
\hline \multirow[b]{2}{*}{ Sector } & \multicolumn{6}{|c|}{ Wind Speed Category, meters/sec } & \multirow[b]{2}{*}{ Total } \\
\hline & $0-2$ & $2-4$ & 4-6 & $6-8$ & 8-12 & $>12$ & \\
\hline $\mathbf{N}$ & 0.00720 & 0.01833 & 0.01159 & 0.00191 & 0.00023 & 0.00000 & 0.03926 \\
\hline NNE & 0.00508 & 0.02159 & 0.01979 & 0.00340 & 0.00009 & 0.00000 & 0.04994 \\
\hline NE & 0.00714 & 0.02587 & 0.02855 & 0.00614 & 0.00026 & 0.00000 & 0.06796 \\
\hline ENE & 0.00654 & 0.02321 & 0.02261 & 0.00500 & 0.00023 & 0.00000 & 0.05759 \\
\hline E & 0.00848 & 0.02296 & 0.01825 & 0.00357 & 0.00003 & 0.00000 & 0.05328 \\
\hline ESE & 0.00911 & 0.01893 & 0.01499 & 0.00217 & 0.00017 & 0.00000 & 0.04537 \\
\hline SE & 0.00877 & 0.01976 & 0.01833 & 0.00063 & 0.00006 & 0.00000 & 0.04754 \\
\hline SSE & 0.00971 & 0.02544 & 0.02590 & 0.00308 & 0.00043 & 0.00000 & 0.06456 \\
\hline $\mathbf{S}$ & 0.00931 & 0.03109 & 0.02835 & 0.00348 & 0.00060 & 0.00000 & 0.07284 \\
\hline ssw & 0.00908 & 0.03578 & 0.02695 & 0.00260 & 0.00074 & 0.00000 & 0.07515 \\
\hline sw & 0.00891 & 0.04140 & 0.03832 & 0.00622 & 0.00049 & 0.00000 & 0.09534 \\
\hline WSW & 0.00740 & 0.03803 & 0.03689 & 0.01348 & 0.00240 & 0.00006 & 0.09825 \\
\hline WSW & 0.00765 & 0.03084 & 0.03541 & 0.01736 & 0.00574 & 0.00014 & 0.09714 \\
\hline WNW & 0.00620 & 0.02264 & 0.02370 & 0.01248 & 0.00545 & 0.00003 & 0.07050 \\
\hline NW & 0.00797 & 0.01596 & 0.01031 & 0.00246 & 0.00100 & 0.00000 & 0.03769 \\
\hline NNW & 0.00648 & 0.01522 & 0.00531 & 0.00051 & 0.00009 & 0.00000 & 0.02761 \\
\hline Total & 0.12500 & 0.40705 & 0.36525 & 0.08449 & 0.01799 & 0.00023 & 1.00000 \\
\hline
\end{tabular}


Table A.13 - Joint Occurrence Frequencies of Wind Direction Sector by Wind Speed Category for the $61 \mathrm{~m}$ Level Central Climatology Tower, Winter 2003

\begin{tabular}{|c|c|c|c|c|c|c|c|}
\hline \multirow[b]{2}{*}{ Sector } & \multicolumn{6}{|c|}{ Wind Speed Category, meters/sec } & \multirow[b]{2}{*}{ Total } \\
\hline & $0-2$ & $2-4$ & $4-6$ & $6-8$ & $8-12$ & $>12$ & \\
\hline $\mathbf{N}$ & 0.01030 & 0.01748 & 0.00891 & 0.00139 & 0.00093 & 0.00000 & 0.03901 \\
\hline NNE & 0.00301 & 0.02083 & 0.01539 & 0.00151 & 0.00012 & 0.00000 & 0.04086 \\
\hline NE & 0.00370 & 0.01933 & 0.02581 & 0.00498 & 0.00023 & 0.00000 & 0.05405 \\
\hline ENE & 0.00370 & 0.02026 & 0.02720 & 0.00706 & 0.00081 & 0.00000 & 0.05903 \\
\hline E & 0.00382 & 0.01586 & 0.01597 & 0.00324 & 0.00000 & 0.00000 & 0.03889 \\
\hline ESE & 0.00347 & 0.01262 & 0.00903 & 0.00220 & 0.00000 & 0.00000 & 0.02732 \\
\hline SE & 0.00440 & 0.00949 & 0.00857 & 0.00058 & 0.00000 & 0.00000 & 0.02303 \\
\hline SSE & 0.00463 & 0.01157 & 0.01493 & 0.00544 & 0.00035 & 0.00000 & 0.03692 \\
\hline S & 0.00417 & 0.01204 & 0.01505 & 0.00764 & 0.00069 & 0.00000 & 0.03958 \\
\hline ssw & 0.00440 & 0.01435 & 0.01921 & 0.00405 & 0.00278 & 0.00000 & 0.04479 \\
\hline sw & 0.00509 & 0.02847 & 0.04063 & 0.01308 & 0.00151 & 0.00000 & 0.08877 \\
\hline WSW & 0.00451 & 0.02662 & 0.05313 & 0.03819 & 0.00498 & 0.00012 & 0.12755 \\
\hline WSW & 0.00532 & 0.03333 & 0.06644 & 0.04502 & 0.02141 & 0.00093 & 0.17245 \\
\hline WNW & 0.00613 & 0.03553 & 0.04491 & 0.02766 & 0.01285 & 0.00012 & 0.12720 \\
\hline NW & 0.00914 & 0.02442 & 0.01088 & 0.00417 & 0.00104 & 0.00000 & 0.04965 \\
\hline NNW & 0.00613 & 0.01794 & 0.00556 & 0.00104 & 0.00023 & 0.00000 & 0.03090 \\
\hline Total & 0.08195 & 0.32014 & 0.38160 & 0.16725 & 0.04792 & 0.00116 & 1.00000 \\
\hline
\end{tabular}

Table A.14 - Joint Occurrence Frequencies of Wind Direction Sector by Wind Speed Category for the $61 \mathrm{~m}$ Level Central Climatology Tower, Spring 2003

\begin{tabular}{|c|c|c|c|c|c|c|c|}
\hline \multirow[b]{2}{*}{ Sector } & \multicolumn{6}{|c|}{ Wind Speed Category, meters/sec } & \multirow[b]{2}{*}{ Total } \\
\hline & $0-2$ & $2-4$ & $4-6$ & $6-8$ & 8-12 & $>12$ & \\
\hline $\mathbf{N}$ & 0.00476 & 0.02061 & 0.00702 & 0.00204 & 0.00000 & 0.00000 & 0.03442 \\
\hline NNE & 0.00532 & 0.02661 & 0.01778 & 0.00419 & 0.00011 & 0.00000 & 0.05401 \\
\hline NE & 0.01019 & 0.02570 & 0.02072 & 0.00215 & 0.00000 & 0.00000 & 0.05876 \\
\hline ENE & 0.00691 & 0.02582 & 0.02842 & 0.00679 & 0.00011 & 0.00000 & 0.06805 \\
\hline E & 0.01313 & 0.02299 & 0.02751 & 0.00996 & 0.00011 & 0.00000 & 0.07371 \\
\hline ESE & 0.00872 & 0.01857 & 0.02299 & 0.00283 & 0.00034 & 0.00000 & 0.05344 \\
\hline SE & 0.00725 & 0.02106 & 0.02559 & 0.00023 & 0.00011 & 0.00000 & 0.05423 \\
\hline SSE & 0.01042 & 0.02095 & 0.02389 & 0.00091 & 0.00000 & 0.00000 & 0.05616 \\
\hline s & 0.01053 & 0.02265 & 0.02638 & 0.00068 & 0.00000 & 0.00000 & 0.06024 \\
\hline ssw & 0.00951 & 0.02695 & 0.02434 & 0.00272 & 0.00011 & 0.00000 & 0.06363 \\
\hline sw & 0.00736 & 0.02491 & 0.04586 & 0.00940 & 0.00034 & 0.00000 & 0.08786 \\
\hline WSW & 0.00634 & 0.03001 & 0.05106 & 0.01495 & 0.00385 & 0.00011 & 0.10632 \\
\hline WSW & 0.00940 & 0.02763 & 0.03782 & 0.01257 & 0.00385 & 0.00000 & 0.09126 \\
\hline WNW & 0.00645 & 0.02740 & 0.02638 & 0.00815 & 0.00113 & 0.00011 & 0.06963 \\
\hline NW & 0.00770 & 0.01857 & 0.01404 & 0.00181 & 0.00034 & 0.00000 & 0.04246 \\
\hline NNW & 0.00566 & 0.01393 & 0.00532 & 0.00079 & 0.00011 & 0.00000 & 0.02582 \\
\hline Total & 0.12964 & 0.37432 & 0.40512 & 0.08016 & 0.01053 & 0.00023 & 1.00000 \\
\hline
\end{tabular}


Table A.15 - Joint Occurrence Frequencies of Wind Direction Sector by Wind Speed Category for the $61 \mathrm{~m}$ Level Central Climatology Tower, Summer 2003

\begin{tabular}{|c|c|c|c|c|c|c|c|}
\hline \multirow[b]{2}{*}{ Sector } & \multicolumn{6}{|c|}{ Wind Speed Category, meters/sec } & \multirow[b]{2}{*}{ Total } \\
\hline & $0-2$ & $2-4$ & $4-6$ & $6-8$ & $8-12$ & $>12$ & \\
\hline $\mathbf{N}$ & 0.00408 & 0.00465 & 0.00113 & 0.00000 & 0.00000 & 0.00000 & 0.00986 \\
\hline NNE & 0.00340 & 0.00635 & 0.00204 & 0.00045 & 0.00000 & 0.00000 & 0.01224 \\
\hline NE & 0.00737 & 0.01213 & 0.00737 & 0.00102 & 0.00011 & 0.00000 & 0.02799 \\
\hline ENE & 0.00635 & 0.01518 & 0.00589 & 0.00091 & 0.00000 & 0.00000 & 0.02833 \\
\hline$E$ & 0.00805 & 0.02663 & 0.00873 & 0.00261 & 0.00000 & 0.00000 & 0.04601 \\
\hline ESE & 0.01167 & 0.02606 & 0.00714 & 0.00227 & 0.00034 & 0.00000 & 0.04748 \\
\hline SE & 0.01099 & 0.02391 & 0.01292 & 0.00034 & 0.00000 & 0.00000 & 0.04816 \\
\hline SSE & 0.01247 & 0.04113 & 0.02516 & 0.00068 & 0.00000 & 0.00000 & 0.07943 \\
\hline S & 0.01269 & 0.06312 & 0.03887 & 0.00227 & 0.00023 & 0.00000 & 0.11717 \\
\hline ssw & 0.01598 & 0.08147 & 0.04623 & 0.00147 & 0.00000 & 0.00000 & 0.14516 \\
\hline sw & 0.01722 & 0.09303 & 0.05088 & 0.00283 & 0.00011 & 0.00000 & 0.16408 \\
\hline WSW & 0.01179 & 0.07513 & 0.03286 & 0.00453 & 0.00068 & 0.00000 & 0.12499 \\
\hline WSW & 0.01043 & 0.04555 & 0.02198 & 0.00238 & 0.00079 & 0.00000 & 0.08113 \\
\hline WNW & 0.00635 & 0.02255 & 0.00691 & 0.00113 & 0.00023 & 0.00000 & 0.03717 \\
\hline NW & 0.00793 & 0.00771 & 0.00215 & 0.00102 & 0.00011 & 0.00000 & 0.01892 \\
\hline NNW & 0.00533 & 0.00567 & 0.00079 & 0.00011 & 0.00000 & 0.00000 & 0.01190 \\
\hline Total & 0.15207 & 0.55025 & 0.27105 & 0.02402 & 0.00261 & 0.00000 & 1.00000 \\
\hline
\end{tabular}

Table A.16 - Joint Occurrence Frequencies of Wind Direction Sector by Wind Speed Category for the 61m Level Central Climatology Tower, Fall 2003

\begin{tabular}{|c|c|c|c|c|c|c|c|}
\hline \multirow[b]{2}{*}{ Sector } & \multicolumn{6}{|c|}{ Wind Speed Category, meters/sec } & \multirow[b]{2}{*}{ Total } \\
\hline & $0-2$ & $2-4$ & 4-6 & $6-8$ & $8-12$ & $>12$ & \\
\hline $\mathbf{N}$ & 0.00802 & 0.02338 & 0.02361 & 0.00241 & 0.00000 & 0.00000 & 0.05742 \\
\hline NNE & 0.00802 & 0.03564 & 0.04366 & 0.00573 & 0.00023 & 0.00000 & 0.09329 \\
\hline NE & 0.00699 & 0.05042 & 0.06131 & 0.01822 & 0.00069 & 0.00000 & 0.13764 \\
\hline ENE & 0.00860 & 0.03736 & 0.03140 & 0.00493 & 0.00012 & 0.00000 & 0.08240 \\
\hline E & 0.00779 & 0.02693 & 0.02235 & 0.00012 & 0.00000 & 0.00000 & 0.05719 \\
\hline ESE & 0.01169 & 0.01857 & 0.01708 & 0.00092 & 0.00000 & 0.00000 & 0.04825 \\
\hline SE & 0.01158 & 0.02132 & 0.02315 & 0.00115 & 0.00012 & 0.00000 & 0.05730 \\
\hline SSE & 0.01123 & 0.02533 & 0.03346 & 0.00642 & 0.00138 & 0.00000 & 0.07781 \\
\hline $\mathbf{S}$ & 0.00951 & 0.02063 & 0.02705 & 0.00206 & 0.00126 & 0.00000 & 0.06051 \\
\hline ssw & 0.00573 & 0.02166 & 0.01731 & 0.00264 & 0.00023 & 0.00000 & 0.04756 \\
\hline sw & 0.00630 & 0.02304 & 0.02006 & 0.00287 & 0.00000 & 0.00000 & 0.05226 \\
\hline WSW & 0.00619 & 0.02223 & 0.01799 & 0.00539 & 0.00023 & 0.00000 & 0.05203 \\
\hline WSW & 0.00596 & 0.01902 & 0.02074 & 0.00963 & 0.00218 & 0.00000 & 0.05753 \\
\hline WNW & 0.00619 & 0.01020 & 0.01478 & 0.01192 & 0.00539 & 0.00000 & 0.04847 \\
\hline NW & 0.00722 & 0.01547 & 0.01135 & 0.00309 & 0.00229 & 0.00000 & 0.03942 \\
\hline NNW & 0.00722 & 0.01616 & 0.00745 & 0.00012 & 0.00000 & 0.00000 & 0.03094 \\
\hline Total & 0.12824 & 0.38735 & 0.39273 & 0.07759 & 0.01410 & 0.00000 & 1.00000 \\
\hline
\end{tabular}

